\title{
PLUTO’s ATMOSPHERE FROM STELLAR OCCULTATIONS IN 2012 AND 2013*
}

\author{
A. Dias-Oliveira ${ }^{1,2}$, B. Sicardy ${ }^{2}$, E. Lellouch ${ }^{2}$, R. Vieira-Martins ${ }^{1,3,4}$, M. Assafin ${ }^{5}$, J. I. B. Camargo ${ }^{1,4}$, F. Braga-Ribas $^{1,6}$, $^{2}$ \\ A. R. Gomes-Júnior ${ }^{5}$, G. Benedetti-Rossi ${ }^{1}$, F. Colas ${ }^{3}$, A. Decock ${ }^{2,7}$, A. Doressoundiram ${ }^{2}$, C. Dumas $^{7}$, M. Emilio $^{8}$, \\ J. Fabrega Polleri ${ }^{9}$, R. Gil-Hutton ${ }^{10,11}$, M. Gillon ${ }^{12}$, J. H. Girard ${ }^{7}$, G. K. T. Hau ${ }^{7}$, V. D. IvanOv ${ }^{13,14}$, E. Jehin $^{12}$, \\ J. LeCACheux $^{2}$, R. Leiva ${ }^{15}$, C. Lopez-Sisterna ${ }^{10}$, L. Mancini ${ }^{16,17}$, J. Manfroid ${ }^{12}$, A. Maury ${ }^{18}$, E. MeZA ${ }^{2,19}$, N. Morales ${ }^{20}$, \\ L. Nagy ${ }^{18}$, C. Opitom ${ }^{12}$, J. L. Ortiz ${ }^{20}$, J. Pollock ${ }^{21}$, F. Roques ${ }^{2}$, C. Snodgrass ${ }^{22}$, J. F. Soulier ${ }^{23}$, A. Thirouin ${ }^{24}$, L. Vanzi ${ }^{15}$, \\ T. WidemanN ${ }^{2}$, D. E. Reichart ${ }^{25}$, A. P. LaCluyze ${ }^{25}$, J. B. Haislip ${ }^{25}$, K. M. Ivarsen ${ }^{2}$, M. Dominik ${ }^{26}$ U. Jørgensen $^{27}$, And \\ J. SKOTTFELT 27,28 \\ ${ }^{1}$ Observatório Nacional/MCTI, Rua General José Cristino 77, Rio de Janeiro-RJ, 20.921-400, Brazil; alexoliveira@on.br \\ ${ }^{2}$ LESIA/Observatoire de Paris, CNRS UMR 8109, Université Pierre et Marie Curie, Université Paris-Diderot, 5 place Jules Janssen, F-92195 Meudon Cédex, France \\ ${ }^{3}$ IMCCE/Observatorie de Paris, 77 Avenue Denfert Rochereau, Paris, F-75014, France \\ ${ }^{4}$ Laboratório Interinstitucional de e-Astronomia-LIneA, Rua General José Cristino, 77, Rio de Janeiro, RJ 20921-400, Brazil \\ ${ }^{5}$ Observatório do Valongo/UFRJ, Ladeira Pedro Antonio 43, Rio de Janeiro-RJ, 20080-090, Brazil \\ ${ }^{6}$ Federal University of Technology-Paraná (UTFPR/DAFIS), R. Sete de Setembro 3165, Curitiba—PR, 80230-901, Brazil \\ ${ }^{7}$ ESO, Alonso de Cordova 3107, Vitacura, 7630355 Santiago, Chile \\ ${ }^{8}$ Observatório Astronômico Departamento de Geociências, Universidade Estadual de Ponta Grossa, Paraná, Brazil \\ ${ }^{9}$ Observatorio Panameño en San Pedro de Atacama (OPSPA), Chile \\ ${ }^{10}$ Complejo Astronómico El Leoncito (CASLEO-CONICET), Av. España 1512 sur, J5402DSP San Juan, Argentina \\ ${ }^{11}$ Universidad Nacional de San Juan, Complejo Universitario "Islas Malvinas," Av. Ignacio de la Roza 590 oeste, J5402DCS-San Juan, Argentina \\ ${ }^{12}$ Institut d'Astrophysique, de Géophysique et Océanographie, Université de Liège, Allée du 6 août 17, B-4000 Liége, Belgium \\ ESO, Karl-Schwarzschild-Str. 2, D-85748 Garching bei, München, Germany \\ ${ }^{14}$ ESO, Alonso de Cordova 3107, Casilla 19001, Santiago 19, Chile \\ ${ }^{15}$ Instituto de Astrofísica, Facultad de Física, Pontificia Universidad Católica de Chile, Av. Vicuña Mackenna 4860, Santiago 7820436, Chile \\ ${ }^{16}$ Max Planck Institute for Astronomy, Königstuhl 17, D-69117, Heidelberg, Germany \\ ${ }^{17}$ INAF-Osservatorio Astrofisico di Torino, via Osservatorio 20, I-10025-Pino Torinese, Italy \\ ${ }^{18}$ San Pedro de Atacama Celestial Explorations (S.P.A.C.E.), San Pedro de Atacama, Chile \\ ${ }^{19}$ Grupo Astronomía, Facultad de Ciencias, Universidad Nacional de Ingienería, Lima, Peru \\ ${ }^{20}$ Instituto de Astrofísica de Andalucía-CSIC, Apt 3004, E-18080, Granada, Spain \\ ${ }^{21}$ Department of Physics and Astronomy, Appalachian State University, Boone, NC 28608, USA \\ 22 Planetary and Space Sciences, Department of Physical Sciences, The Open University, Milton Keynes, MK7 6AA, UK \\ ${ }^{23}$ Association Des Étoiles pour Tous, 19 Rue Saint Laurent, Maisoncelles, F-77320 Saint Martin du Boschet, France \\ ${ }^{24}$ Lowell Observatory, 1400 W Mars Hill Rd, Flagstaff, Arizona, USA \\ ${ }^{25}$ Department of Physics and Astronomy, University of North Carolina-Chapel Hill, North Carolina 27599, USA \\ ${ }^{26}$ SUPA, University of St Andrews, School of Physics \& Astronomy, North Haugh, St Andrews, KY16 9SS, UK \\ ${ }^{27}$ Niels Bohr Institute, University of Copenhagen, Juliane Maries vej 30, DK-2100 Copenhagen Ø, Denmark \\ ${ }^{28}$ Centre for Electronic Imaging, Department of Physical Sciences, The Open University, Milton Keynes, MK7 6AA, UK \\ Received 2015 June 22; accepted 2015 August 10; published 2015 September 18
}

\begin{abstract}
We analyze two multi-chord stellar occultations by Pluto that were observed on 2012 July 18th and 2013 May 4th, and respectively monitored from five and six sites. They provide a total of fifteen light curves, 12 of which were used for a simultaneous fit that uses a unique temperature profile, assuming a clear (no haze) and pure $\mathrm{N}_{2}$ atmosphere, but allowing for a possible pressure variation between the two dates. We find a solution that satisfactorily fits (i.e., within the noise level) all of the 12 light curves, providing atmospheric constraints between $\sim 1190 \mathrm{~km}$ (pressure $\sim 11 \mu \mathrm{bar}$ ) and $\sim 1450 \mathrm{~km}$ (pressure $\sim 0.1 \mu \mathrm{bar}$ ) from Pluto's center. Our main results are: (1) the best-fitting temperature profile shows a stratosphere with a strong positive gradient between $1190 \mathrm{~km}$ (at $36 \mathrm{~K}$, $11 \mu \mathrm{bar})$ and $r=1215 \mathrm{~km}(6.0 \mu \mathrm{bar})$, where a temperature maximum of $110 \mathrm{~K}$ is reached; above it is a mesosphere with a negative thermal gradient of $-0.2 \mathrm{~K} \mathrm{~km}^{-1}$ up to $\sim 1390 \mathrm{~km}(0.25 \mu \mathrm{bar})$, where the mesosphere connects itself to a more isothermal upper branch around $81 \mathrm{~K}$; (2) the pressure shows a small (6\%) but significant increase ( $6 \sigma$ level) between the two dates; (3) without a troposphere, Pluto's radius is found to be $R_{\mathrm{P}}=1190 \pm 5 \mathrm{~km}$. Allowing for a troposphere, $R_{\mathrm{P}}$ is constrained to lie between 1168 and $1195 \mathrm{~km}$; and (4) the currently measured CO abundance is too small to explain the mesospheric negative thermal gradient. Cooling by HCN is possible, but only if this species is largely saturated. Alternative explanations like zonal winds or vertical compositional variations of the atmosphere are unable to explain the observed mesospheric negative thermal gradient.
\end{abstract}

Key words: methods: data analysis - methods: observational - planets and satellites: atmospheres - planets and satellites: physical evolution - planets and satellites: terrestrial planets - techniques: photometric

\section{INTRODUCTION}

* Partly based on observations made with the ESO camera NACO at the Very Large Telescope (Paranal), under program IDs 089.C-0314(C) and 291.C5016. The prediction uses observations made with the WFI camera at the $2.2 \mathrm{~m}$ Telescope, under program ID 079.A-9202(A).
Stellar occultations are a very powerful tool for discovering and studying, among other things, tenuous atmospheres around remote bodies. Pluto's atmosphere was discovered using this technique (Hubbard et al. 1988; Elliot et al. 1989; 

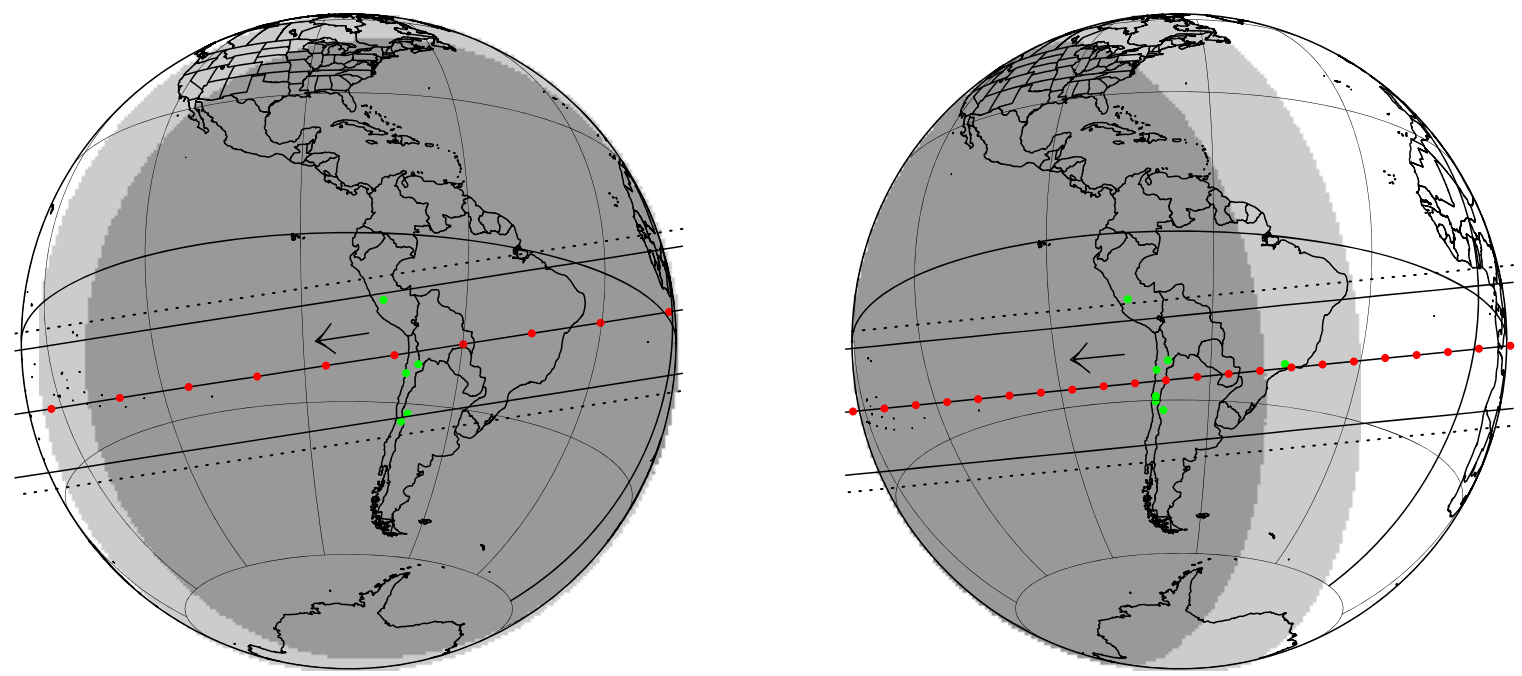

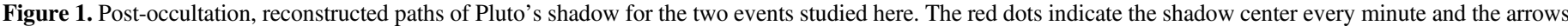

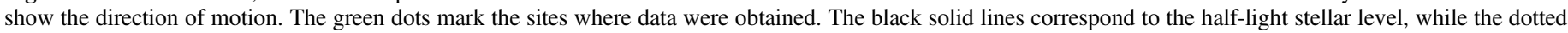

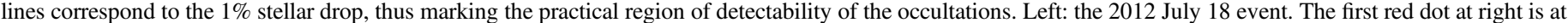

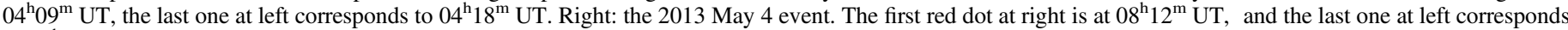
to $08^{\mathrm{h}} 33^{\mathrm{m}} \mathrm{UT}$.

Brosch 1995), and its spectacular twofold expansion between 1988 and 2003 was also revealed using stellar occultations (Elliot et al. 2003; Sicardy et al. 2003). Other trans-Neptunian objects were explored with this technique and so far none of them have exhibited atmospheres at the 10 nbar pressure level (that is, three orders of magnitude smaller than for Pluto). This includes Charon (Sicardy et al. 2006), Eris (Sicardy et al. 2011), Makemake (Ortiz et al. 2012), and Quaoar (Braga-Ribas et al. 2013).

All of those bodies have sizes and surface gravities that are comparable to those of Pluto, within a factor of two. As such, the derived upper limits constrain the physical conditions necessary for the appearance and maintenance of atmospheres around a body with a given ice composition and heliocentric distance.

Here we analyze results derived from two Pluto stellar occultations (2012 July 18 and 2013 May 04) that provide signal-to-noise ratios $(\mathrm{S} / \mathrm{Ns})$ that are among the best ever obtained during such events. They are furthermore combined with well-sampled multi-chord coverages, providing a good absolute radial scale for the atmosphere extension.

We use the simplest possible model, assuming a spherically symmetric, clear (no haze), pure $\mathrm{N}_{2}$ atmosphere with a constant temperature both horizontally and with time. Our model satisfactorily fits 12 of the selected light curves and provides accurate density, pressure, and temperature profiles for radii between $1190 \mathrm{~km}$ (11 $\mu$ bar pressure level) and $1450 \mathrm{~km}$ ( $\sim 0.1 \mu$ bar) from Pluto's center, while also providing constraints on Pluto's radius.

As Pluto's atmospheric pressure is dominated by the vapor equilibrium pressure at its surface, it is very sensitive to tiny changes of temperature and the available amount of exposed ice. This induces strong seasonal effects over the Plutonian year (Hansen \& Paige 1996) that can be monitored and analyzed through stellar occultations (Young 2013). In that context, our data reveal a small but significant increase of pressure between 2012 and 2013, which can be used for constraining current Pluto seasonal models (see Olkin et al. 2015 for a detailed analysis).
Our results are obtained in the context of the forthcoming flyby of the dwarf planet by the NASA New Horizons spacecraft in 2015 July. Consequently, they can be used as a basis of comparison with the New Horizons findings.

\section{THE 2012 AND 2013 PLUTO STELLAR OCCULTATIONS}

\subsection{Predictions}

From astrometric observations along Pluto's path onto the sky plane between 2008 and 2015, performed at the European Southern Observatory (ESO)'s $2.2 \mathrm{~m}$ telescope, Assafin et al. (2010) made accurate predictions for stellar occultations involving the dwarf planet and its satellites.

In this context, the two occultations analyzed here, one on 2012 July 18 and the other on 2013 May 04, stood out as promising events, owing to the magnitudes of the candidate stars and to the presence of several potential observing sites along the shadow path.

Follow-up astrometric observations of the stars were carried out in order to improve the predictions. These observations were made with the $1.6 \mathrm{~m}$ (Perkin-Elmer) and $0.6 \mathrm{~m}$ (Boller \& Chivens) telescopes, at Pico dos dias Observatory (OPD, IAU code 874), and they are done wherever possible within our access time.

Moreover, 16 positive detections of other occultations by Pluto, which occured between 2005 and 2013, were used to improve Pluto's ephemeris offset (see Benedetti-Rossi et al. 2014 for details).

Days before the event, we carried out observations with Pluto and the occulted star present in the same field of view of our charge-coupled devices (CCDs) in order to minimize systematic biases like catalog errors.

\subsection{Observations}

The 2012 July 18 Pluto occultation was observed near its zenith from five sites in South America (Figure 1). The circumstances and technical details of the observations are 
Table 1

Circumstances of the 2012 July 18 Pluto Occultation

\begin{tabular}{|c|c|c|c|c|}
\hline Site & $\begin{array}{l}\text { Lat. (d:m:s) } \\
\text { Lon. (d:m:s) } \\
\text { alt (m) }\end{array}$ & $\begin{array}{c}\text { Telescope } \\
\text { Instrument/Filter }\end{array}$ & Exp. Time/Cycle (s) ${ }^{\mathrm{a}}$ & Observers \\
\hline $\begin{array}{l}\text { Observatory UC } \\
\text { (Santa Martina) }\end{array}$ & $\begin{array}{c}33: 16: 09.0 \mathrm{~S} \\
70: 32: 04.0 \mathrm{~W} \\
1450\end{array}$ & $\begin{array}{c}0.4 \mathrm{~m} \\
\mathrm{CCD} / \text { clear }\end{array}$ & $1.0 / 1.0$ & R. Leiva Espinoza \\
\hline Cerro Burek & $\begin{array}{c}31: 47: 12.4 \mathrm{~S} \\
69: 18: 24.5 \mathrm{~W} \\
2591\end{array}$ & $\begin{array}{c}\text { ASH }^{\mathrm{b}} 0.45 \mathrm{~m} \\
\text { SBIG-STL11000/clear }\end{array}$ & $13.0 / 15.7$ & N. Morales \\
\hline Paranal & $\begin{array}{c}24: 37: 31.0 \mathrm{~S} \\
70: 24: 08.0 \mathrm{~W} \\
2635\end{array}$ & $\begin{array}{c}\text { VLT Yepun } 8.2 \mathrm{~m} \\
\mathrm{NACO} / \mathrm{H}\end{array}$ & $0.2 / 0.2$ & J. Girard \\
\hline $\begin{array}{l}\text { San Pedro } \\
\text { de Atacama }\end{array}$ & $\begin{array}{c}22: 57: 12.3 \mathrm{~S} \\
68: 10: 47.6 \mathrm{~W} \\
2397\end{array}$ & $\begin{array}{c}\text { ASH2 } 0.4 \mathrm{~m} \\
\text { SBIG-STL11000/clear }\end{array}$ & $13.0 / 15.44$ & N. Morales \\
\hline Huancayo & $\begin{array}{c}12: 02: 32.2 \mathrm{~S} \\
75: 19: 14.7 \mathrm{~W} \\
3344\end{array}$ & $\begin{array}{c}0.2 \mathrm{~m} \\
\mathrm{CCD} / \text { clear }\end{array}$ & $\begin{array}{c}10.24 / 10.24^{\mathrm{c}} \\
5.12 / 5.12^{\mathrm{c}}\end{array}$ & E. Meza \\
\hline
\end{tabular}

Notes.

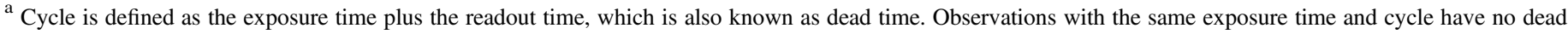
time.

b ASH-Astrograph for the Southern Hemisphere.

${ }^{c}$ Exposure time was changed at 04:11:46 UT.

provided in Table 1. The 2013 May 04 event was recorded from six sites, under similar conditions (Figure 1), providing ten light curves (Table 2). Various astrometric, photometric, and physical parameters associated with each event are summarized in Table 3.

Figure 2 displays the reconstructed geometries of each event, showing the Plutocentric latitudes and altitudes probed by the primary stellar image at each site; see the Appendix for details. For Paranal (2012 July 18), for the sake of illustration we plot the trajectories of both primary and secondary stellar images. As we shall mention later, the contribution of the secondary image is small but not negligible compared to that of the primary image near the shadow center. Note that in the ingress, the primary image probes the hemisphere that is in summer conditions, and at the egress the image probes the hemisphere that is in winter conditions.

\subsection{Photometry and Calibration}

Classical bias, dark, flat-field, and sky subtraction provide the occultation light curves displayed in Figures 3-5. In all cases, a reference star brighter that is than the target was used to correct for low-frequency sky-transparency variations.

As expected, the best $\mathrm{S} / \mathrm{N}$ light curve was obtained at Paranal on 2012 July 18, using the NAOS-CONICA ${ }^{28}$ (NACO; Lenzen et al. 2003; Rousset et al. 2003) camera attached to the $8.2 \mathrm{~m}$ "Yepun" Very Large Telescope (VLT) of the ESO, in open-loop (without adaptive optics), at a continuous rate of 5 frames per second (achieved windowing the detector in "cube mode" as in Girard et al. 2010) in the $H$-band. Moreover, this is the only data set for which we have an accurate photometric calibration, which allows us to subtract the contribution of Pluto and Charon from the occultation light curve (see below). As such, the 2012 July 18 data provide the best constraints on

\footnotetext{
28 NAOS-CONICA is Nasmyth Adaptive Optics System (NAOS) and NearInfrared Imager and Spectrograph (CONICA).
}

Pluto's atmospheric structure. However, on average the 2013 May 04 light curves have better S/Ns than those of 2012 July 18 , as well as a better spatial sampling, thus providing better constraints on the absolute vertical scale of the atmosphere.

Calibration images were taken with NACO some 20 minutes before the 2012 event. They show resolved images of Pluto, Charon, and the star under excellent seeing conditions (Figure 6). Digital coronagraphy (Assafin et al. 2008, 2009) was used to remove the star contamination from Pluto and Charon images. Classical aperture photometry finally provided the Pluto + Charon flux relative to the occulted star. This allows us to estimate the residual stellar flux in the deepest part of the 2012 July 18 occultation at Paranal, with a value that varied from $2.3 \pm 0.8 \%$ to $1.8 \pm 0.8 \%$ of its unocculted value in the central part of the occultation (Figure 7).

\section{MODELING OF PLUTO'S ATMOSPHERE}

The general idea for modeling Pluto's atmosphere is to use an iterative procedure, combining both direct ray-tracing and inversion approaches. We first invert our best $\mathrm{S} / \mathrm{N}$ light curve to retrieve Pluto's atmospheric density, pressure, and temperature profiles (see the Appendix and Vapillon et al. 1973). The retrieved temperature profile is then used as a guide to generate, through direct ray-tracing, synthetic occultation light curves that are simultaneously fitted to all of the observed light curves obtained at a given date. This pins down the location of Pluto's shadow center relative to the occultation chords for both the 2012 and 2013 events (Figure 2). Finally, the inversion of the best light curve is performed again and the procedure is resumed. This iterative process eventually provides the accurate geometry of each event, as well as consistent density, pressure, and temperature profiles that best fit all of the occultation light curves.

Simplifying assumptions are made in our procedure (possible caveats are discussed later): (i) Pluto and its atmosphere are spherically symmetric, and all quantities 
Table 2

Circumstances of the 2013 May 04 Pluto Occultation

\begin{tabular}{|c|c|c|c|c|}
\hline Site & $\begin{array}{l}\text { Lat. (d:m:s) } \\
\text { Lon. (d:m:s) } \\
\text { alt meters }\end{array}$ & $\begin{array}{c}\text { Telescope } \\
\text { Instrument/Filter }\end{array}$ & Exp. Time/Cycle (s) & Observers \\
\hline Cerro Burek & $\begin{array}{c}31: 47: 12.4 \mathrm{~S} \\
69: 18: 24.5 \mathrm{~W} \\
2591 \mathrm{~m}\end{array}$ & $\begin{array}{c}\text { ASH } 0.45 \mathrm{~m} \\
\text { SBIG-STL11000/clear }\end{array}$ & $6 / 8$ & $\begin{array}{l}\text { J.L. Ortiz } \\
\text { N. Morales }\end{array}$ \\
\hline $\begin{array}{l}\text { CASLEO } \\
\text { (Leoncito) }\end{array}$ & $\begin{array}{c}31: 47: 55.6 \mathrm{~S} \\
69: 17: 44.9 \mathrm{~W} \\
2,492 \mathrm{~m}\end{array}$ & $\begin{array}{c}\text { Jorge Sahade } 2.15 \mathrm{~m} \\
\mathrm{CCD} / \mathrm{R}\end{array}$ & $5 / 6.8$ & $\begin{array}{l}\text { R. Gil-Hutton } \\
\text { C. Lopez-Sisterna }\end{array}$ \\
\hline Cerro Tololo & $\begin{array}{c}\text { 30:10:03.4 S } \\
70: 48: 19.0 \mathrm{~W} \\
2,207\end{array}$ & $\begin{array}{l}\text { PROMPT }^{\mathrm{a}} 0.4 \mathrm{~m} \\
\text { P1, P3, P4, P5 } \\
\text { CCD } / \text { clear }\end{array}$ & $\begin{array}{c}5 / 8 \\
\text { P3 offset } 2 \mathrm{~s} \\
\text { P4 offset } 4 \mathrm{~s} \\
\text { P5 offset } 6 \mathrm{~s}\end{array}$ & J. Pollock \\
\hline La Silla & $\begin{array}{c}291521.276 \mathrm{~S} \\
704420.184 \mathrm{~W} \\
2,336\end{array}$ & $\begin{array}{c}\text { Danish } 1.54 \mathrm{~m} \\
\text { Lucky Imager } / \mathrm{Z}(\lambda>650 \mathrm{~nm} \\
\mathrm{CCD} / \text { iXon response })\end{array}$ & $\begin{array}{c}0.1 / 0.1 \\
\text { Several } \\
\text { interruptions } \\
\text { due to image } \\
\text { cube writing }\end{array}$ & L. Mancini \\
\hline La Silla & $\begin{array}{c}291516.59 \mathrm{~S} \\
704421.82 \mathrm{~W} \\
2,315\end{array}$ & $\begin{array}{l}\text { TRAPPIST }^{\mathrm{b}} 0.6 \mathrm{~m} \\
\text { CCD/clear }\end{array}$ & $4.5 / 6$ & $\begin{array}{l}\text { E. Jehin, } \\
\text { A. Decock, M. Gillon } \\
\text { C. Opitom }\end{array}$ \\
\hline Pico dos Dias & $\begin{array}{c}223207.8 \mathrm{~S} \\
453457.7 \mathrm{~W} \\
1,811\end{array}$ & $\begin{array}{l}\mathrm{B} \& \mathrm{C}^{\mathrm{c}} 0.6 \mathrm{~m} \\
\quad \mathrm{CCD} / \mathrm{I}\end{array}$ & $5 / 5.40$ & $\begin{array}{l}\text { M. Assafin, } \\
\text { A. Ramos-Gomes Jr }\end{array}$ \\
\hline Ponta Grossa & $\begin{array}{c}250524.00 \mathrm{~S} \\
500936.00 \mathrm{~W} \\
909\end{array}$ & $\begin{array}{l}\text { Meade } 160.4 \mathrm{~m} \\
\mathrm{CCD} / \text { clear }\end{array}$ & $\begin{array}{c}5 \\
\text { Technical } \\
\text { Problems }\end{array}$ & M. Emilio \\
\hline Cerro Paranal & $\begin{array}{c}24: 37: 31.0 \mathrm{~S} \\
70: 24: 08.0 \mathrm{~W} \\
2635\end{array}$ & $\begin{array}{c}\text { UT4 Yepun } 8.2 \mathrm{~m} \\
\mathrm{NACO} / \mathrm{H}\end{array}$ & $0.2 / 0.2$ & G. Hau \\
\hline \multirow[t]{5}{*}{$\begin{array}{l}\text { San Pedro } \\
\text { de Atacama }\end{array}$} & $\begin{array}{c}22: 57: 12.3 \mathrm{~S} \\
68: 10: 47.6 \mathrm{~W} \\
2397\end{array}$ & $\begin{array}{c}\text { Caisey } 0.5 \mathrm{~m} \mathrm{f} / 8 \\
\mathrm{CCD} / \mathrm{V}\end{array}$ & $3 / 4.58$ & A. Maury \\
\hline & $\cdots$ & $\begin{array}{c}\text { Caisey } 0.5 \mathrm{~m} \mathrm{f} / 6.8 \\
\mathrm{CCD} / \mathrm{B}\end{array}$ & $4 / 4.905$ & L. Nagy \\
\hline & $\cdots$ & $\begin{array}{c}\mathrm{CAO}^{\mathrm{d}} 0.4 \mathrm{~m} \\
\mathrm{CCD} / \mathrm{R}\end{array}$ & $4 / 6.35$ & J.F. Soulier \\
\hline & $\cdots$ & ASH2 $0.4 \mathrm{~m}$ & $\begin{array}{c}\text { STL11000 } \\
\text { Technical problem }\end{array}$ & N. Morales \\
\hline & $\cdots$ & $\begin{array}{l}\text { OPSPA }^{\mathrm{e}} 0.3 \mathrm{~m} \\
\mathrm{CCD} / \text { clear }\end{array}$ & $5 / 11.1$ & J. Fabrega Polleri \\
\hline Huancayo & $\begin{array}{c}12: 02: 32.2 \mathrm{~S} \\
75: 19: 14.7 \mathrm{~W} \\
3344\end{array}$ & $\begin{array}{c}\text { Meade } 80.2 \mathrm{~m} \\
\mathrm{CCD} / \text { clear }\end{array}$ & $\begin{array}{l}10.24 / 10.24 \\
\text { Negative chord } \\
\text { (no occultation) }\end{array}$ & E. Meza \\
\hline
\end{tabular}

Notes.

${ }^{a}$ PROMPT: Panchromatic Robotic Optical Monitoring and Polarimetry Telescopes.

b Jehin et al. (2011).

${ }^{\mathrm{c}}$ B\&C: Boller \& Chivens.

${ }^{\mathrm{d}}$ CAO: Campo Catino Observatory.

${ }^{\mathrm{e}}$ OPSPA: Observatorio Panameño en San Pedro de Atacama. 
Table 3

Parameters of the Two Occultations

\begin{tabular}{|c|c|c|}
\hline & 2012 July 18 & 2013 May 04 \\
\hline Star coordinates ${ }^{\mathrm{a}}$ & $\begin{array}{l}\alpha=18^{\mathrm{h}} 32^{\mathrm{m}} 14^{\mathrm{s}} \cdot 6720 \\
\delta=-19^{\mathrm{d}} 24^{\prime} 19^{\prime \prime} \cdot 295\end{array}$ & $\begin{array}{c}\alpha=18^{\mathrm{h}} 47^{\mathrm{m}} 52^{\mathrm{s}} .5322 \\
\delta=-19^{\mathrm{d}} 41^{\prime} 24^{\prime \prime} 3738\end{array}$ \\
\hline Ephemeris & DE413/PLU022 & DE413/PLU031 \\
\hline Pluto geocentric distance & $4.68244 \times 10^{9} \mathrm{~km}($ at $04: 13 \mathrm{UT})$ & $4.76882 \times 10^{9} \mathrm{~km}($ at $08: 23 \mathrm{UT})$ \\
\hline Sub-observer and sub-solar latitudes ${ }^{\mathrm{b}}$ & $B=+47.10$ days, $B^{\prime}=+47.54$ days & $B=+49.95$ days, $B^{\prime}=+48.64$ days \\
\hline Pluto's north pole position angle ${ }^{\mathrm{b}}$ & $P=-56.88$ days & $P=-52.91$ days \\
\hline Shadow velocity & $\approx 23.0 \mathrm{~km} \mathrm{~s}^{-1}$ & $\approx 10.6 \mathrm{~km} \mathrm{~s}^{-1}$ \\
\hline Magnitudes $^{c}$ & $V=14.7, R=13.7, K=10.9$ & $V=14.1, R=14.0, K=12.4$ \\
\hline
\end{tabular}

Notes.

a J2000, UCAC2 system.

${ }^{\mathrm{b}}$ Assuming Pluto's north pole position (J2000) of Tholen et al. (2008): $\alpha_{\mathrm{p}}=08^{\mathrm{h}} 52^{\mathrm{m}} 12^{\mathrm{s}} \cdot 94, \delta_{\mathrm{p}}=-06^{\mathrm{d}} 10^{\prime} 04{ }^{\prime \prime} 8$.

${ }^{\mathrm{c}}$ From the NOMAD catalog (Zacharias et al. 2004).
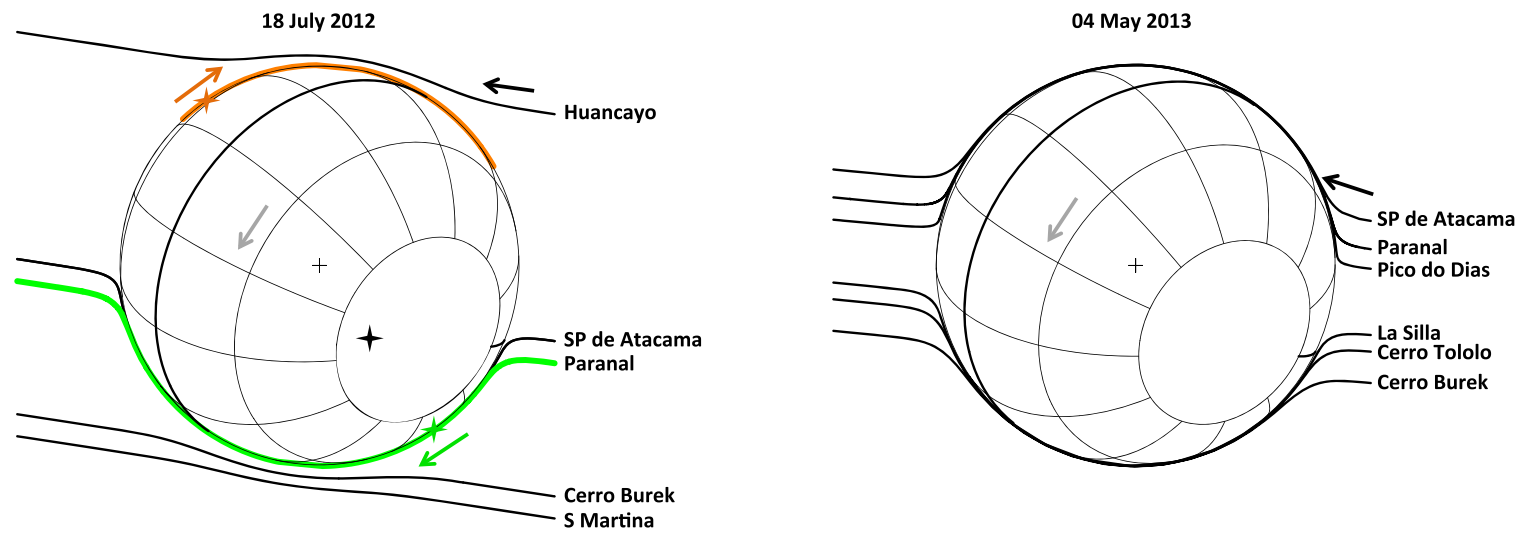

Figure 2. Left: the trajectories of the primary stellar images relative to Pluto, as seen from the five stations used on 2012 July 18 ; see Table 1. The black arrow shows the general direction of stellar motion. Here, Pluto's stellar motion has an assumed radius of $R_{\mathrm{P}}=1190 \mathrm{~km}$ (see the text), and its center is indicated by the cross symbol. The gray arrow inside the disk indicates the direction of rotation. In the case of Paranal, we have plotted the path of the primary image in green, and the associated path of the secondary image in orange (see also Figure 7). The green and orange arrows show the corresponding local stellar motion along Pluto's limb. Note that the two images move in opposite directions. The black star symbol shows the star position as seen from Paranal at a given, arbitrary moment, while the green and orange star symbols indicate the associated primary and secondary images at that time, respectively. Note that the three star symbols and the cross are aligned. Right: the same as the left panel for the 2013 May 04 occultation, with only the paths of the primary stellar images plotted. In both panels, the summer, with Pluto's hemisphere permanently lit, is at right, and the low-insolation winter limb is at left.

depend only on the radius $r$ (defined as the distance to Pluto's body center). (ii) The atmosphere is transparent (no haze present). (iii) It is an ideal gas in hydrostatic equilibrium; in our case, it is a pure molecular nitrogen $\mathrm{N}_{2}$ atmosphere, neglecting other minor species like methane. (iv) Moreover, we assume that $T(r)$ is time-independent, i.e., the temperature profiles are the same in 2012 and 2013. Once $T(r)$ is derived, as detailed later, the density and pressure profiles $n(r)$ and $p(r)$ are derived from the hydrostatic and ideal gas equations (Equation (5)) once a boundary condition is provided, i.e., the pressure at a given radius. (v) Although $T(r)$ is taken as time-independent, the pressure is not. This is justified by the fact that the pressure is very sensitive to Pluto's surface temperature through the vapor pressure equilibrium equation. For instance, a $1 \mathrm{~K}$ temperature increase at the surface results in a twofold increase of pressure or so (Figure 8). Thus, the pressure is a free parameter in our fits. More precisely, Equation (5) requires a boundary condition once $T(r)$ is fixed. So we use the pressure $p_{r}$ at an arbitrary radius $r$ as a free parameter. We choose $r=1275 \mathrm{~km}$ for an easier comparison with other works that provide the pressure at that level (see, e.g., Olkin et al. 2015). This level corresponds to a normalized stellar flux of $\approx 0.45$ in the shadow plane. Once $p_{1275}$ is given, the density and pressure profiles $n(r)$ and $p(r)$ are uniquely defined.

We choose the 2012 July 18 occultation light curve obtained at VLT/NACO to perform the first inversion. We use this particular light curve because it has the highest $\mathrm{S} / \mathrm{N}$ of all (Figure 3), and also because this is the only one for which we have a reliable measurement of the background contribution from Pluto and Charon (Figure 7), which is necessary to correctly invert any occultation light curve. The successive steps of our procedure are as follows:

(1) The inversion reveals a strong increase of temperature just above the surface (stratosphere), followed by a turning point where the temperature reaches a maximum (stratopause), then a region with a mild negative gradient (mesosphere), and finally an isothermal upper branch (see the Appendix and Figure 13). Using the prescriptions described by Equations (4), we adjust the coefficients $c_{1}, \ldots c_{9}$, controlling the profile $T(r)$ in order to best-fit the inverted temperature profiles (see Table 4).

(2) Keeping the profile $T(r)$ fixed in shape, we simultaneously fit seven of the light curves obtained on 2013 May 04. The free parameters of that fit are the two coordinates defining the shadow center, the pressure $p_{1275}$ at radius $1275 \mathrm{~km}$, and the value of $r_{1}$, the deepest point that we consider in our profile. 
18 July 2012

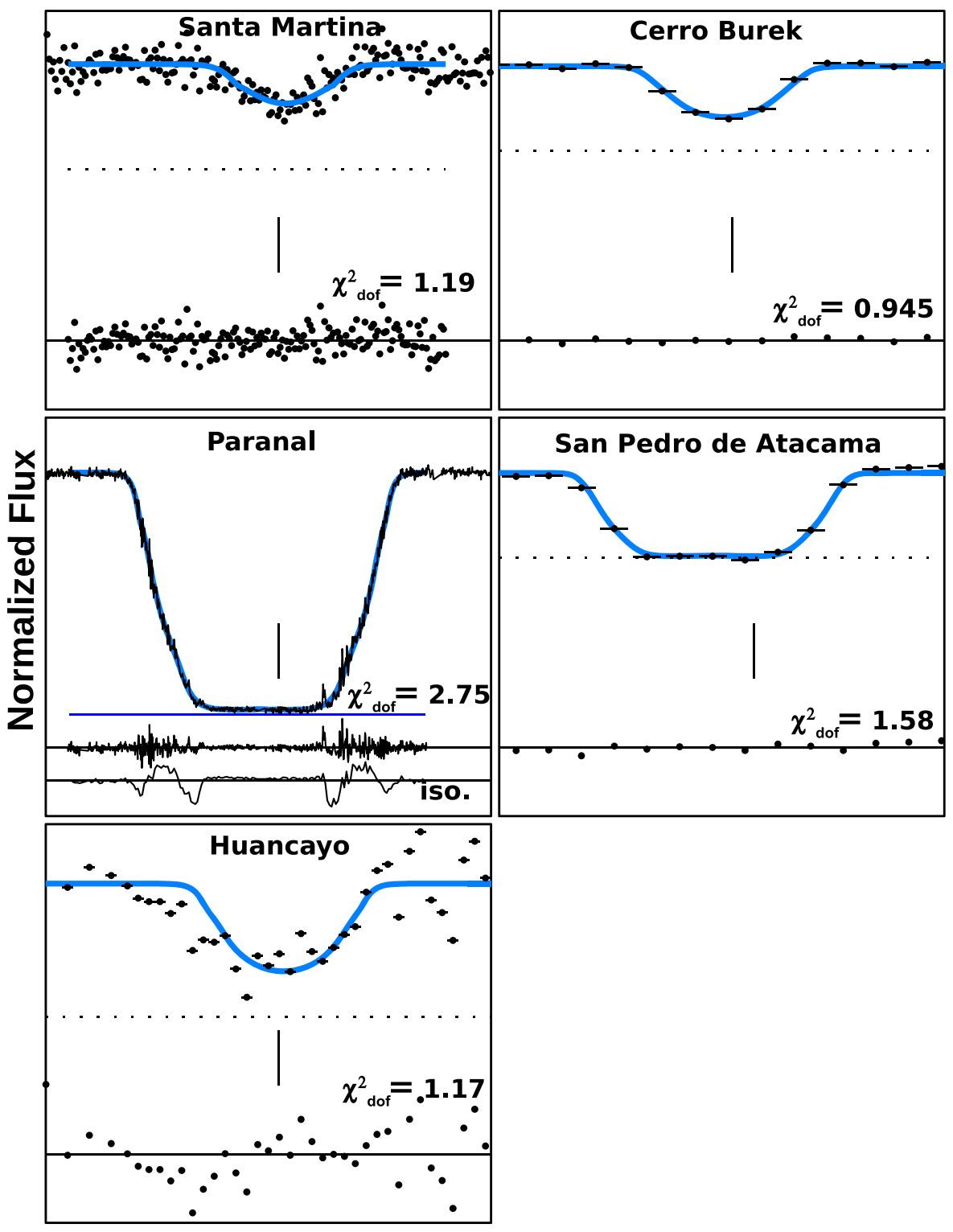

Time

Figure 3. Blue curves are a simultaneous fit to the 2012 July 18 light curves, using the best atmospheric model described in Table 4 and Figure 13 . The number at the lower right of each panel is the value of $\chi_{\text {dof }}^{2}$ (Equation (11)), i.e., the $\chi^{2}$ per degree of freedom for each corresponding fit. Each panel spans 3 minutes of data, with the vertical tick marks located at 04:13 UT. All of the light curves show the total flux (star+Pluto+Charon) plotted at the same vertical scale. The horizontal bars on the Cerro Burek, San Pedro de Atacama, and Huancayo data points represent the respective integration times. The zero flux is indicated by the solid horizontal line at the bottom of each panel, together with the residuals (data minus model). The dotted horizontal lines mark the fitted zero stellar fluxes (or equivalently, the Pluto+Charon contribution to the total flux), obtained using our best Pluto atmospheric model. The blue horizontal line in the Paranal panel marks the measured zero stellar flux at that station, the only one at which a photometric calibration was possible (see the text and Figures 6 and 7). In the Paranal panel, we have also added the residuals (labeled "iso.") obtained by forcing an isothermal mesosphere at $T_{\text {iso }}=95.5 \mathrm{~K}$. The residuals have been averaged over $5 \mathrm{~s}$ time intervals and shifted vertically by -0.12 to better show the clear discrepancy between the isothermal mesospheric model and the data. Other values chosen for $T_{\text {iso }}$ would result in the same qualitative behavior. In essence, isothermal mesospheres do not provide satisfactory fits to the NACO light curve.

At this stage, when $r_{1}$ is varied, all of the other radii, $r_{2}, r_{3}$, and $r_{4}$, defining $T(r)$ (see Equation (4)), are changed by the same amount. In other words, the entire profile $T(r)$ is vertically displaced by this amount. Thus, $r_{1}$ eventually fixes the absolute vertical scale of the atmospheric profile. Note that $r_{1}$ is not, a priori, the radius of the stratobase, nor Pluto's surface radius. In practice, the choice of $r_{1}$ is made so that the stellar rays from the faint secondary image passing at $r_{1}$ have a contribution to the total flux that is negligible compared to the light curve noise level. Thus, taking larger values of $r_{1}$ would create artificial discontinuities in the synthetic light curve, while smaller values would require useless computation time. To find Pluto's shadow center, we separate the fit along the direction of the star motion relative to Pluto from the fit perpendicular to that direction. This is because the fit along the star motion is essentially independent of the atmospheric model and is generally more accurate than the fit perpendicular to that direction.

Note that the 2013 light curves generally have a better S/N than those of 2012 (excluding the VLT data set) because of a 
04 May 2013

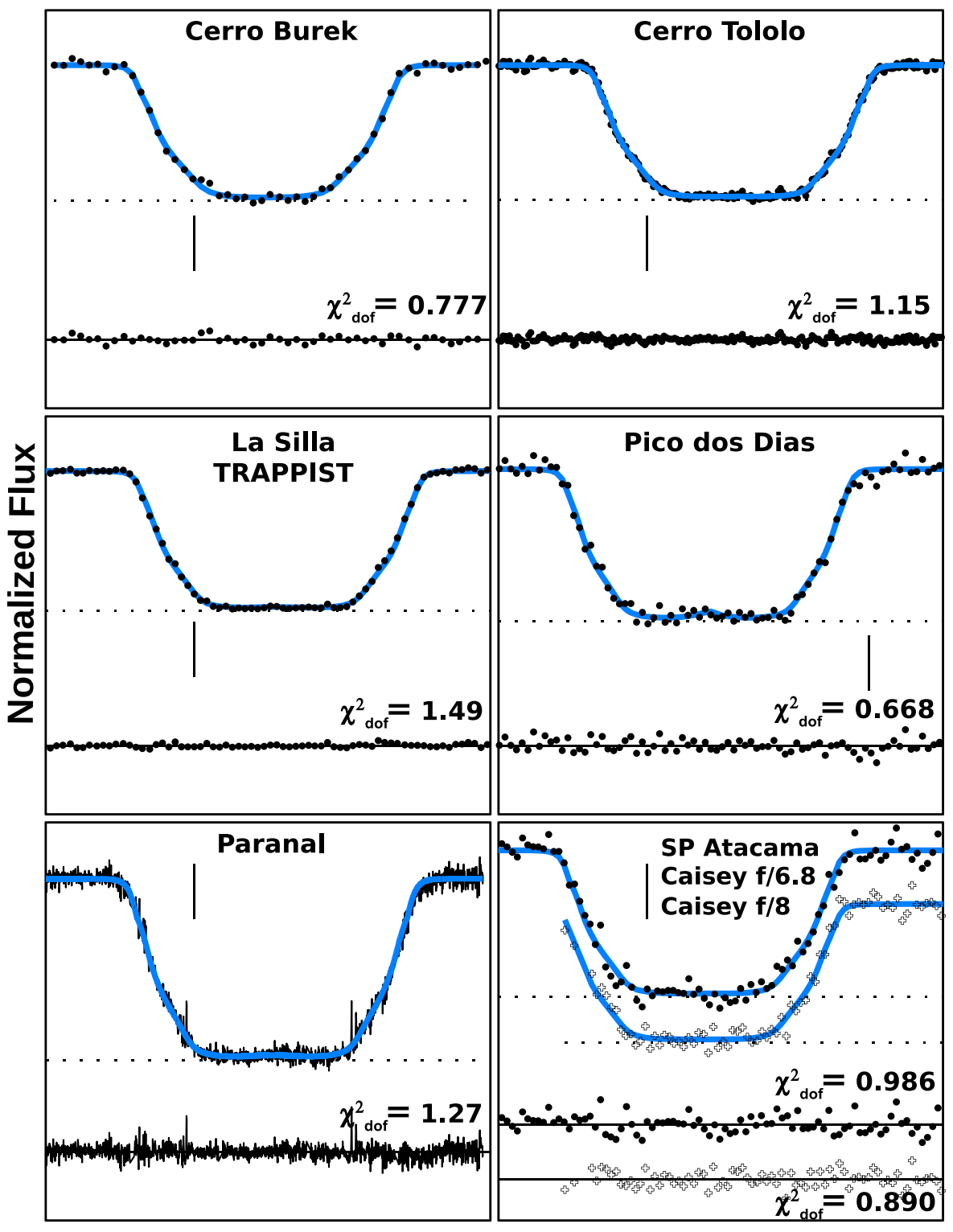

Time

Figure 4. Same as Figure 3 for the 2013 May 04 event. Each panel now represents 6 minutes of data, with the vertical tick mark located at 08:22 UT. Note that the two light curves from San Pedro ("SP") de Atacama have been displaced vertically by \pm 0.1 for better viewing.

better distribution of the chords (Figure 2). Consequently, the 2013 occultation light curves provide a better constraint for $r_{1}$, or equivalently, for the absolute vertical scale of the atmospheric model, than those of 2012.

(3) Fixing $r_{1}$ to its value found in step (2), we turn back to the 2012 July 18 data set and simultaneously fit the five corresponding light curves, varying Pluto's shadow center and $p_{1275}$.

The procedure is then resumed at point (1). It is a converging process that provides consistent solutions for the shape of the profile $T(r)$, the absolute vertical scale for $T(r)$, the centers of Pluto's shadow for both events, and the two boundary conditions $p_{1275}$ for the 2012 July 18 and 2013 May 04 events. This fitting procedure has a total of 12 free parameters: the nine coefficients $c_{1}, \ldots c_{9}$, the two coordinates that define Pluto's shadow center, and the pressure $p_{1275}$.
As commented before, the 2012 July 18 NACO light curve is the only one for which the Pluto + Charon contribution is measured (Figure 7). Thus, the stellar flux was normalized between that value and the full unocculted flux before starting the fit procedure.

For the other light curves, the inverse approach was used: the background Pluto + Charon flux was imposed by linearly adjusting the normalized, synthetic stellar flux to the actual occultation light curve, through a least-squares fit. As the residual stellar flux well inside the shadow is mainly controlled by the density scale height of the deep stratosphere (Equation (15)), this means that the structure of that region is in fact dominated by the NACO, 2012 July 18 data. The other light curves thus mainly serve to constrain the atmospheric structure above that level (mesosphere). 


\section{May 2013}

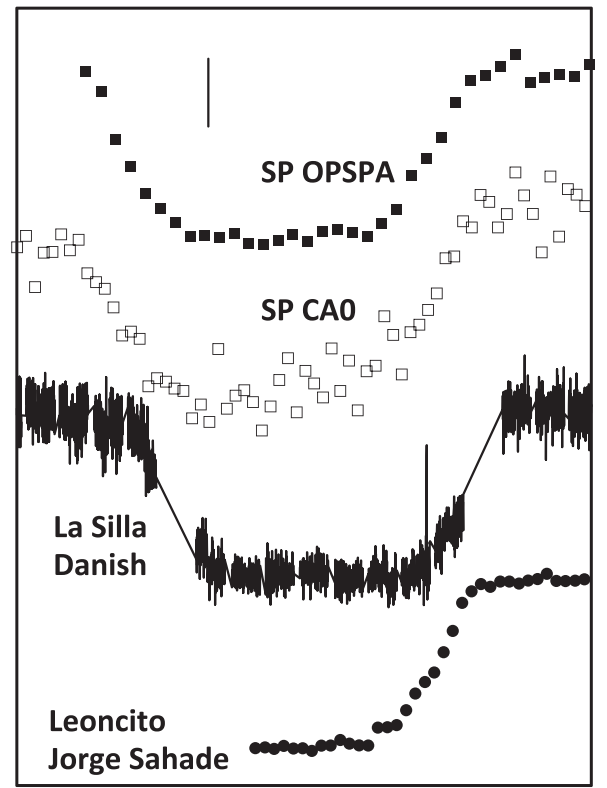

Figure 5. Same as Figure 4, but for the light curves that were not included in the fit, either due to lower $\mathrm{S} / \mathrm{N}$, or interruptions during the acquisition. See Table 2 for instrumental details ("SP" refers to San Pedro de Atacama and acronyms refer to telescope used in that station.). Note that the Leoncito, Danish, and SP light curves duplicate the observations of the Cerro Burek, La Silla TRAPPIST, and Caisey telescopes, respectively.

\section{GENERAL ATMOSPHERIC STRUCTURE}

The best fits of our synthetic light curves to the data are shown in Figures 3 and 4. For each light curve, the residuals are displayed at the bottom of the corresponding panel. They show that a unique global model satisfactorily explains all of the observations, with $\chi^{2}$ values per degree of freedom $\left(\chi_{\text {dof }}^{2}\right.$; see Equation (11)) close to unity, except for the 2012 July 18 NACO data (Figures 3 and 4). In fact, due to the quality of this particular data set, the residuals are dominated by spikes associated with wave activity, as illustrated in Figure 7. The wave activity, including the one observed in the NACO data, is discussed in detail elsewhere (see French et al. 2015).

The parameters of the best atmospheric model are listed in Table 4. Note that the only parameter that differs between 2012 July 18 and 2013 May 04 is the boundary condition, i.e., the pressure $p_{1275}$ at $r=1275 \mathrm{~km}$. Table 4 reveals a small $(6 \%)$ but significant $(6 \sigma$ level $)$ increase of pressure, from $p_{1275}=$ $2.16 \pm 0.02 \mu$ bar in 2012 July to $p_{1275}=2.30 \pm 0.01 \mu$ bar in 2013 May, corresponding to an average pressure increase rate of $7.5 \%$ per year.

Based on various occultation data collected, Young (2013) and Olkin et al. (2015) report a general pressure increase of some 3.5\%-7.5\% per year between 2006 and 2013, consistent with our result above. Note that our value of $p_{1275}=2.30 \pm 0.01 \mu$ bar for 2013 May differs from Olkin et al.'s (2015) result $(2.70 \pm 0.2 \mu$ bar) by a barely significant $0.4 \pm 0.2 \mu$ bar. Part of this difference could be due to the different methods used to derive those numbers, as Olkin et al. (2015) use an isothermal fit to the upper part of the light curves, while we use a combination of the mesosphere with a negative thermal gradient and an upper isothermal branch (Figure 13).

Figure 8 displays the density versus radius, the temperature versus pressure, the temperature versus radius, and the temperature gradient of our best model. Also shown superimposed in that figure are the ingress and egress profiles retrieved from the inversions of the 2012 July 18 NACO light curve, respectively corresponding to the summer and winter hemispheres, as far as the primary stellar image is concerned. Figure 9 is a more detailed view of the bottom of the temperature and temperature gradient profiles, close to Pluto's surface.

The shaded areas in Figures 8 and 9 indicate the $1 \sigma$ error envelopes caused by (i) the photometric noise in the NACO light curve, which mainly affects the upper parts of the profiles, and (ii) the uncertainty on the Pluto + Charon contribution to the total observed flux, which mainly affects the lower parts of the profiles. The methods for calculating these uncertainty domains are described in the Appendix. The temperature profiles are furthermore affected by another source of uncertainty, namely (iii) the a priori unknown temperature boundary condition, inherent to the nature of Equation (5) (a first order differential equation). As examples, we show in Figure 8 (gray lines in panels (b)-(d)) the profiles obtained by changing by $\pm 5 \mathrm{~K}$ the nominal boundary condition $(T=80.5 \mathrm{~K}$ at $r=1390 \mathrm{~km})$ of the egress, inverted NACO temperature profile.

Both the photometric noise and the ignorance of the temperature boundary condition cause an exponential divergence of the uncertainty domain for $T$ and $d T / d r$ as $r$ increases, with an $e$-folding distance equal to the density scale height $H$ (Equation (14) and Figure 8). Nevertheless, we note that if we have independent information on Pluto's atmosphere, e.g., from theoretical models or forthcoming observations from the New Horizons mission, then we can constrain our temperature at rather high altitudes. For instance, at radius $r=1450 \mathrm{~km}$ (pressure $\sim 0.1 \mu \mathrm{bar}$ ), the $1 \sigma$ uncertainty on $T$ caused by photometric noise is about $\pm 2.5 \mathrm{~K}$. Conversely, the two alternative solutions $T(r)$ given as examples in Figure 8 (the gray lines in panel (c)), using different boundary conditions, differ from each other by $30 \mathrm{~K}$ at that same radius. Consequently, they can be distinguished well above the noise level if we dispose of independent constraints on the thermal properties of the atmosphere at that radius.

For instance, the warmer gray profile with strong positive temperature gradient in Figure 8 can be discarded if we adopt current models that predict that UV heating is efficient only at much higher levels (Zhu et al. 2014). The same is true for the cooler gray temperature profile in panel (c) of Figure 8: it shows a negative gradient in the $1400-1450 \mathrm{~km}$ range that is too strong, considering that atmospheric escape may cause temperatures as low as $\sim 60 \mathrm{~K}$, but only much higher in the atmosphere (Zhu et al. 2014).

Figure 8 reveals three regions in our thermal profile, from bottom to top: a stratosphere with a strong positive gradient that starts around $1190 \mathrm{~km}$, with a temperature near $36 \mathrm{~K}$ and a pressure $11 \mu \mathrm{bar}$, and reaches a maximum temperature of $110 \mathrm{~K}$ at the stratopause (near $r=1215 \mathrm{~km}, 6.0 \mu \mathrm{bar}$ ). It then follows a mesosphere with a mild negative thermal gradient of $-0.2 \mathrm{~K} \mathrm{~km}^{-1}$ up to the mesopause $(r \sim 1390 \mathrm{~km}, 0.25 \mu \mathrm{bar})$, where it connects itself to a more isothermal upper part around $81 \mathrm{~K}$. These regions are now described in detail.

\section{STRATOSPHERE}

As explained in the Appendix, the residual stellar flux in the mid-part of the occultation is proportional to the local density 

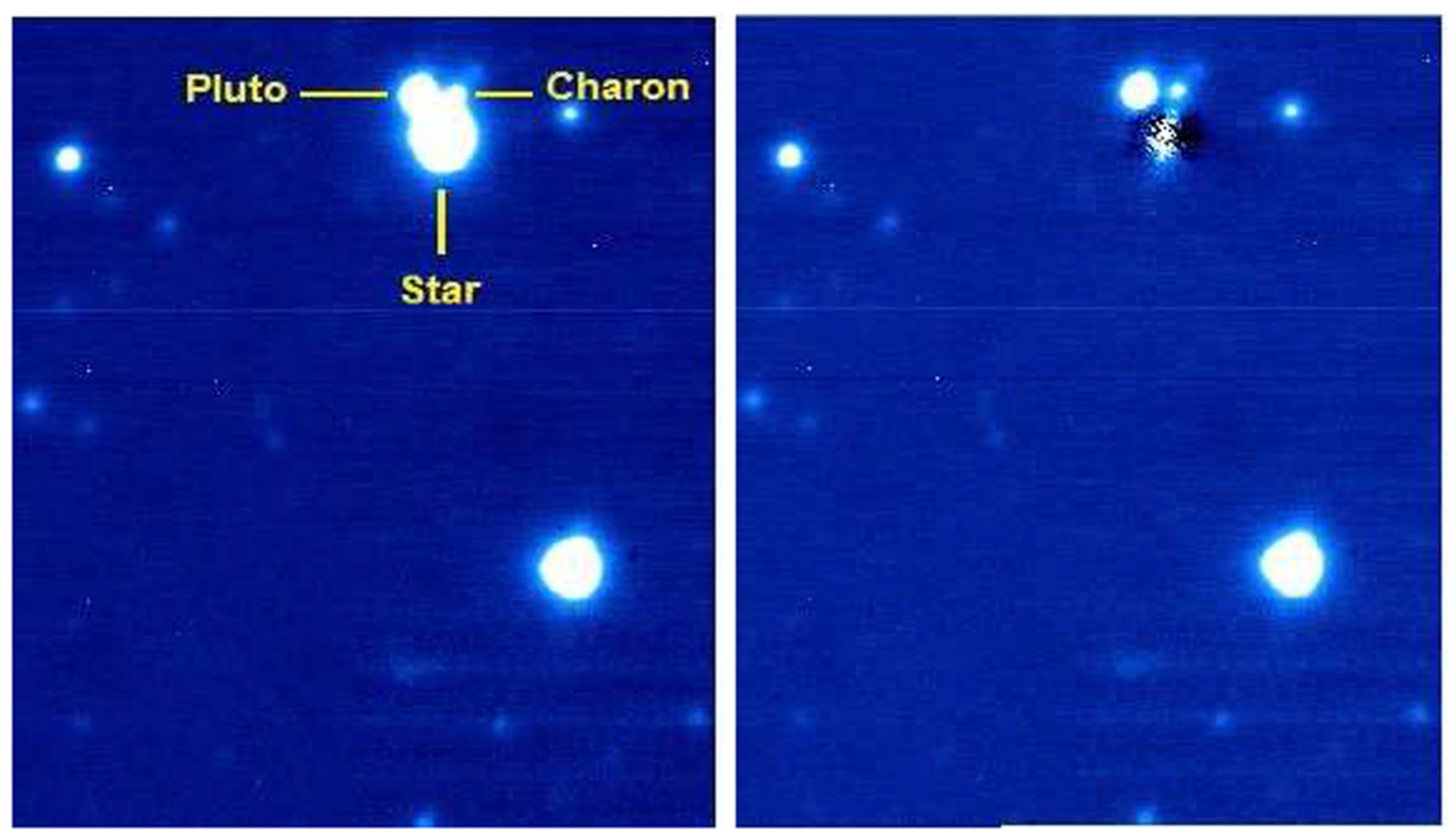

Figure 6. Photometric calibration of the 2012 July 18 event (Paranal/VLT, NACO H-band). Left: image taken some 20 minutes before the event, showing the small separation between Pluto, Charon, and the star $(\sim 1$ "). Right: the same image after a digital coronagraphy treatment that removed the stellar image. See the text for details.

scale height $H$, which is itself related to the strong stratospheric temperature gradient (Equation (15)).

It is important to note that at its closest approach to Pluto's shadow center on 2012 July 18, our model predicts that the secondary image observed at Paranal contributes by $20 \%$ to the total, primary + secondary stellar residual flux (Figure 7). This is not negligible and explains why we have to extend our raytracing model below the deepest radius obtained for the inverted temperature profiles (red and blue lines in Figure 9). In fact, the inversion procedure assumes that there is only one (primary) stellar image contributing to the flux at any moment, while the direct ray-tracing procedure does account for the presence of the two images. When the secondary image appears and disappears (at the extremities of the orange trajectory shown in Figure 2), it reaches the radius $r_{1}=1190.4 \mathrm{~km}$ (Table 4). Its appearance and disappearance cause small discontinuities in the synthetic flux, but they are too small to be distinguished from the noise (Figure 7).

Due to the uncertainty on the Pluto + Charon flux contribution, the deepest point of our model is determined to be at $1190 \pm 5 \mathrm{~km}$ (Figure 9). At that point, nitrogen reaches its saturation vapor pressure (Figure 8), and thus condenses in principle into ice, i.e., it reaches Pluto's surface. In that context, we obtain a solution with a clear nitrogen atmosphere and a Pluto radius of $1190 \pm 5 \mathrm{~km}$, which consistently explains all of our observations, accounting for the presence of both the primary and secondary images.

Other models are possible though. Based on a more incomplete and lower quality data set than used here, Lellouch et al. (2009) conclude that the nitrogen condensation level occurs somewhere in the range 1187-1197 km, consistent with the present work. However, a shallow adiabatic troposphere with dry or wet nitrogen (or methane) may exist below $1190 \mathrm{~km}$. Nevertheless, there is little freedom for such tropospheric models because (i) they tend to create caustics in the light curves that are not observed and (ii) they provide a cold methane column density that would be detected by other means. More precisely, using spectral data, Lellouch et al. (2009) find possible tropospheric solutions in a narrow region of the parameter space, with depths that cannot exceed $17 \mathrm{~km}$. Similarly, again combining constraints from spectra with a preliminary analysis of the occultation data presented in this work, Lellouch et al. (2015a) concluded that Pluto's radius should be between $1180-1188 \mathrm{~km}$, some $2-8 \mathrm{~km}$ below the condensation radius of $1190 \mathrm{~km}$ derived above.

That said, we assume here that the atmosphere is haze-free, a subject of debate since the discovery of Pluto's atmosphere. Analyzing a high S/N occultation observed in 2006, Young et al. (2008) conclude that a haze-only explanation for the light curve is extremely unlikely. In fact, the clear-atmosphere model implies a temperature profile that naturally connects the maximum temperature of $\sim 110 \mathrm{~K}$ near $1215 \mathrm{~km}$ to the surface at average temperature of $\sim 50 \mathrm{~K}$ (Lellouch et al. 2000, 2013; see Figure 8).

Other constraints come from a central flash observation during a stellar occultation in 2007 July. From that event, Olkin et al. (2014) conclude that the flash is consistent with a transparent atmosphere with a temperature gradient of $5 \mathrm{~K} \mathrm{~km}^{-1}$ at $1196 \mathrm{~km}$, fully consistent with our results (Figure 9). Olkin et al. (2014) exclude in particular a hazeonly model to explain the central flash, although combinations of a thermal gradient and a haze mechanism are possible. In the same vein, Gulbis et al. (2015) use a wavelength-resolved occultation from 2011 to constrain the presence of hazes in Pluto's atmosphere. Although haze models do improve the fit residuals, a clear atmosphere with a steep thermal gradient at the bottom is also consistent with the observations.

Finally, we note that the residual stellar flux exhibits a significant decrease in the bottom of the light curve, from $2.3 \%$ to $1.8 \%$ of its unocculted value, in the central part of the occultation as observed from Paranal on 2012 July 18 

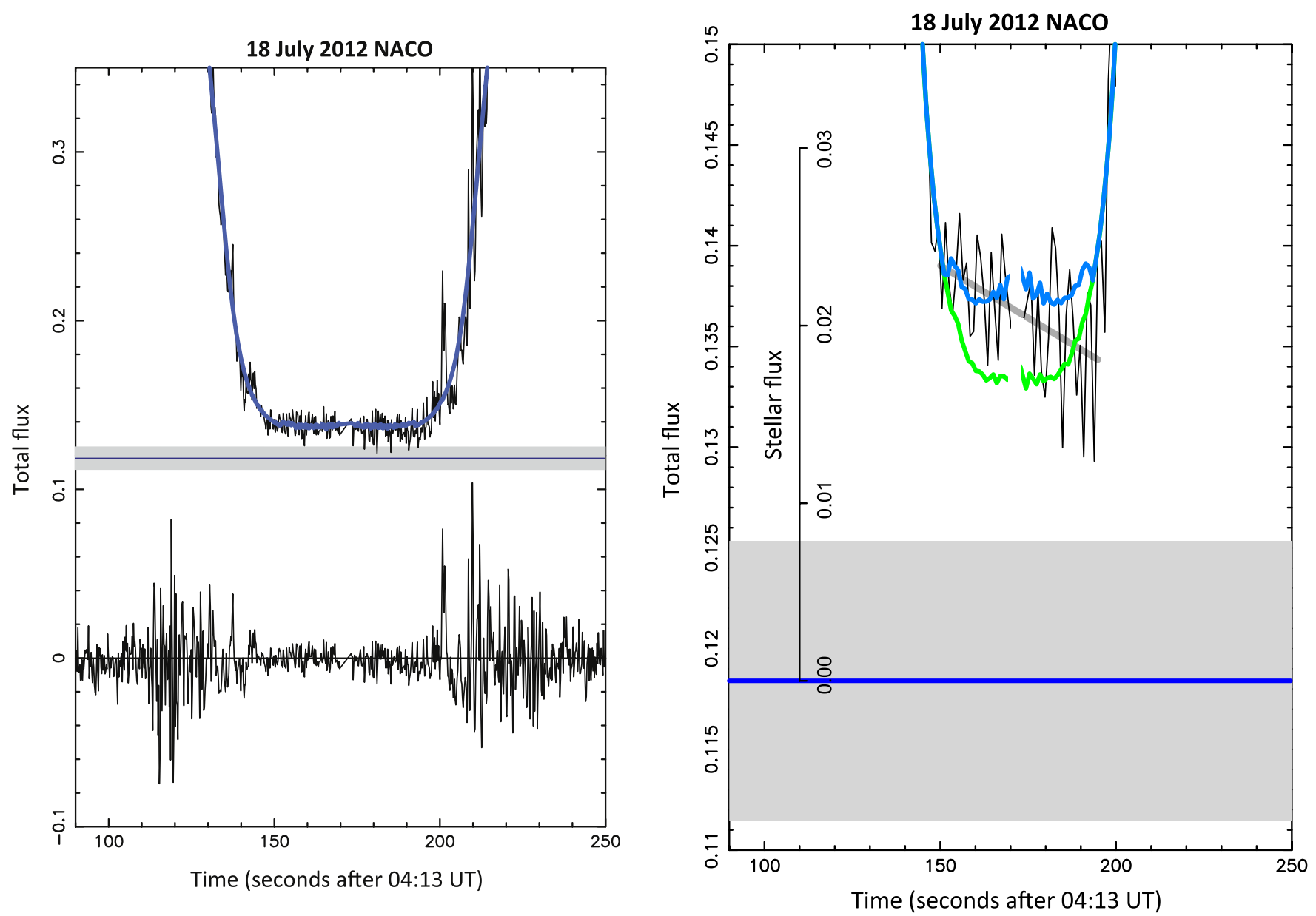

Figure 7. Left: details of the fit to the NACO 2012 July 18 light curve (see also the middle left panel of Figure 3). The horizontal blue line in the gray shaded area indicates the Pluto + Charon contribution to the total observed flux and its $1 \sigma$ error bar, $0.1184 \pm 0.007$. The residuals curve at the bottom clearly shows the spike activity at ingress and egress. Right: expanded view of the left panel. The data have been binned over $1 \mathrm{~s}$ time intervals to better show the flux decrease during the central phase of the occultation. The flux of the expected primary stellar image is plotted in green, while the blue curve is the sum of the primary and secondary images, according to the model (see Figure 2 and the Appendix). Thus, the contribution of the secondary image is the difference between the blue and green curves. Note that the interruption of data acquisition (about $3 \mathrm{~s}$ ) at mid-occultation, which is necessary for the writing of the data cube before the start of the next data cube. The inclined gray line is a linear fit to the central part of the light curve, which illustrates the ingress/egress asymmetry of the residual stellar flux. The vertical axis inside the box at left indicates the value of the residual, normalized stellar flux. It shows that the stellar flux decreased from about $2.3 \%$ to about $1.8 \%$ of its full unocculted value during that interval. The systematic error on those values is $\pm 0.8 \%$ (corresponding to the shaded area).

(Figure 7). This behavior was already pointed out by Sicardy et al. (2003), based on another high S/N occultation observed in 2002 August. In both cases, the residual stellar flux decreased as the primary stellar image first scanned the summer, permanently lit northern lower atmosphere, and then the winter, low-insolation region (Figure 2). This point is discussed in the last section.

\section{MESOSPHERIC NEGATIVE TEMPERATURE GRADIENT}

Above the stratopause $(r \sim 1215 \mathrm{~km})$, the temperature profile exhibits a negative temperature gradient up to $r \sim 1390 \mathrm{~km}$, with an average value of $d T / d r \sim-0.2$ $\mathrm{K} \mathrm{km}^{-1}$. In this $\sim 170 \mathrm{~km}$ radius interval, the temperature decreases by some $30 \mathrm{~K}$. This mesospheric gradient is affected very little by the choice of the temperature boundary condition (see Figure 8, panel (d)). While the photometric noise and the boundary condition problem induce rapidly diverging solutions for $d T / d r$ above $\sim 1400 \mathrm{~km}$, the thermal gradient between $\sim 1250 \mathrm{~km}$ and $\sim 1360 \mathrm{~km}$ is robustly constrained around
$-0.2 \mathrm{~K} \mathrm{~km}^{-1}$, with a typical fine-scale scatter of $\pm 0.05 \mathrm{~K} \mathrm{~km}^{-1}$ that is dominated by Pluto's wave activity, and not by the photometric noise. In this interval, the thermal gradient remains smaller (in absolute value) than the dry adiabatic lapse rate $-g / c_{\mathrm{p}}$ (Figure 8), where $g$ is the acceleration of gravity (Equation (6)) and $c_{\mathrm{p}}=1.04 \times 10^{3}$ $\mathrm{J} \mathrm{K}^{-1} \mathrm{~kg}^{-1}$ is the specific heat at constant pressure for $\mathrm{N}_{2}$. Thus, the mesosphere remains convectively stable.

Note that in principle we may choose an extreme temperature boundary condition that provides an isothermal mesosphere, i.e., a thermal profile that is much warmer than the warmer gray profile shown in panel (c) of Figure 8. As commented earlier, however, this replaces one problem with another one, namely that the upper part of our profile is too warm, with seemingly no plausible physical explanation.

The negative mesospheric thermal gradient is further confirmed by the inversions of our best $\mathrm{S} / \mathrm{N}$ light curves obtained in 2012 July and 2013 May (see Figure 10). This eliminates random, low-frequency sky-transparency variations that may have corrupted the light curves. Moreover, such gradients have also been reported in previous, independent 

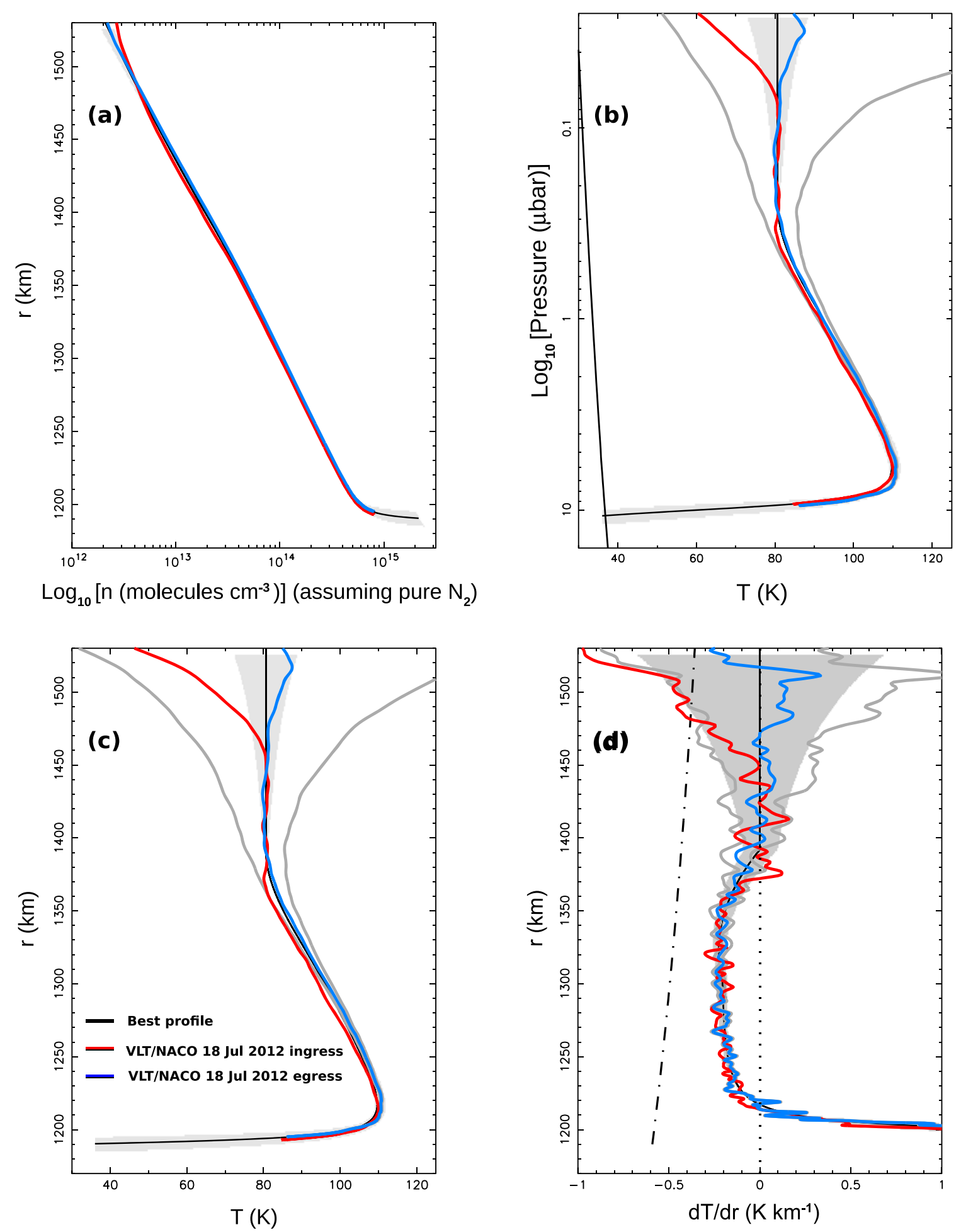

Figure 8. In all of the panels, the black solid line is the model that best fits all of the 2012 July 18 NACO light curves (see Figure 3 and Table 4 ). The red (resp. blue) lines are the particular profiles obtained from the inversion of the NACO 2012 July 18 light curve at ingress/summer (resp. egress/winter). The shaded areas at the top of the profiles indicate the expected $\pm 1 \sigma$ fluctuations caused by the photometric noise ( see the text). The shaded areas at the bottom of the profiles are the $\pm 1 \sigma$ uncertainty domain caused by the uncertainty on the Pluto + Charon contribution to the 2012 July 18 NACO light curve (see Figure 7). (a) Molecular density vs. radius (assuming a pure $\mathrm{N}_{2}$ atmosphere); (b) temperature vs. pressure; (c) temperature vs. radius; (d) temperature gradient vs. radius. The two gray temperature profiles in panels (b), (c), and (d) show the effect of different temperature boundary conditions for the egress NACO profile. More precisely, those profiles differ from the nominal one (black lines) by $\pm 5 \mathrm{~K}$ at $1390 \mathrm{~km}$. The oblique solid line at the left of panel (b) is the vapor pressure equilibrium limit for $\mathrm{N}_{2}$ (Fray \& Schmitt 2009). Nitrogen should condense to the left of this line so that its intersection with the temperature profile may define Pluto's surface in the absence of a troposphere ( see Figure 9 and the text for details). The dash-dotted line in panel (d) is the dry adiabat for a pure $\mathrm{N}_{2}$ atmosphere. 
Table 4

Pluto Atmospheric Model

\begin{tabular}{ll}
\hline \hline & Physical Parameters \\
Pluto's mass $^{\mathrm{a}}$ & $G M=8.703 \times 10^{11} \mathrm{~m}^{3} \mathrm{~s}^{-2}$ \\
$\begin{array}{c}\text { Nitrogen molecular } \\
\text { mass }\end{array}$ & $\mu=4.652 \times 10^{-26} \mathrm{~kg}$ \\
$\begin{array}{c}\text { Nitrogen molecular } \\
\text { refractivity }\end{array}$ & $K=1.091 \times 10^{-23}+\left(6.282 \times 10^{-26} / \lambda_{\mu \mathrm{m}}^{2}\right)$ \\
Boltzmann constant & $k=1.380626 \times 10^{-23} \mathrm{~J} \mathrm{~K}^{-1}$ \\
\hline
\end{tabular}

The Nine Free Parameters of the Best Temperature Profile ${ }^{\mathrm{d}}$

\begin{tabular}{ll}
\hline$r_{1}, T_{1}, d T / d r\left(r_{1}\right)$ & $1190.4 \pm 1 \mathrm{~km}, 36 \mathrm{~K}, 16.9 \mathrm{~K} \mathrm{~km}^{-1}$ \\
$r_{2}, T_{2}$ & $1217.3 \mathrm{~km}, 109.7 \mathrm{~K}$ \\
$r_{3}, T_{3}$ & $1302.4 \mathrm{~km}, 95.5 \mathrm{~K}$ (implying \\
& $\left.d T / d r\left(r_{3}\right)=-0.206 \mathrm{~K} \mathrm{~km}^{-1}\right)$ \\
$r_{4}, T_{4}$ & $1392.0 \mathrm{~km}, 80.6 \mathrm{~K}$ \\
\hline$c 1, c 2$ & $1.41397736 \times 10^{-3}, 2.59861886 \times 10^{-3}$ \\
$c 3, c 4$ & $-2.19756021 \times 10^{-6},-4.81764971 \times 10^{-7}$ \\
$c 5, c 6$ & $8.66619700 \times 10^{-8},-3.6213609 \times 10^{4}$ \\
$c 7, c 8$ & $8.2775269 \times 10^{1},-6.27372563 \times 10^{-2}$ \\
$c 9$ & $1.58068760 \times 10^{-5}$ \\
\hline
\end{tabular}

$c 9$

\begin{tabular}{lcc}
\multicolumn{3}{c}{ The Three Free Parameters Particular to Each Event } \\
\hline & 2012 July 18 & 2013 May 04 \\
\hline $\begin{array}{l}\text { Pressure at } r=1275 \mathrm{~km}, \\
p_{1275}\end{array}$ & $2.16 \pm 0.02 \mu \mathrm{bar}$ & $2.30 \pm 0.01 \mu \mathrm{bar}$ \\
$\begin{array}{c}\text { Time of closest geo- } \\
\text { centric approach }\end{array}$ & $04: 13: 37.24 \pm 0.07 \mathrm{UT}$ & $08: 22: 27.11 \pm 0.09 \mathrm{UT}$ \\
$\begin{array}{c}\text { Distance of closest geo- } \\
\text { centric approach }\end{array}$ & $-404.6 \pm 2.7 \mathrm{~km}$ & $-723.5 \pm 2.7 \mathrm{~km}$ \\
\hline
\end{tabular}

Notes.

${ }^{\mathrm{a}}$ Tholen et al. (2008).

${ }^{b}$ Assumed to be the only constituent in the ray-tracing code (see the text).

${ }^{c}$ Washburn (1930, p. 11). For both NACO observations of 2012 and 2103, the $H$-band $(\lambda=1.6 \mu \mathrm{m})$ was used.

${ }^{\mathrm{d}}$ Or equivalently, the nine coefficients $c 1, \ldots c 9$; see the text and Figure 13 for the definition of the various quantities given here.

${ }^{\mathrm{e}}$ So that there are twelve free parameters for each date.

${ }^{\mathrm{f}}$ Distance of Pluto's center to the star at the closest approach, projected in the sky plane, as seen from the geocenter. A negative value means that Pluto's center went south of the star in the sky plane.

works. For instance Young et al. (2008) derive and discuss a $d T / d r=-0.086 \pm 0.033 \mathrm{~K} \mathrm{~km}^{-1}$ gradient at $r=1275 \mathrm{~km}$ from the 2006 June 12 occultation, while Elliot et al. (2007) give $-0.17 \pm 0.05 \mathrm{~K} \mathrm{~km}^{-1}$ for the same occultation and at the same radius. Gulbis et al. (2015) report a gradient of $-0.23 \pm 0.05 \mathrm{~K} \mathrm{~km}^{-1}$ in the $1310-1450 \mathrm{~km}$ region from the 2011 June 23 occultation, consistent with Person et al. (2013) for that event. Finally, Bosh et al. (2015) derive values of $-0.17 \pm 0.03$ and $-0.24 \pm 0.01 \mathrm{~K} \mathrm{~km}^{-1}$ around $1280-1300 \mathrm{~km}$ for occultations observed on 2012 September 09 and 2013 May 04, respectively.

The origin of this thermal gradient is still debated. Two classes of possible explanations can be proposed: (1) the presence of cooling minor species and (2) yet unmodeled physical mechanisms. They are now examined in detail.

\subsection{Possible Cooling by $\mathrm{CO}$ or $\mathrm{HCN}$}

Radiative-conductive models of Pluto's atmosphere were initially developed by Yelle \& Lunine (1989), Hubbard et al.
(1988), Lellouch (1994), and Lellouch et al. (2015a) to explain the recently discovered gross characteristics of Pluto's atmosphere: a large lower atmosphere temperature gradient and a warmer $(\sim 100 \mathrm{~K})$ mesosphere. These studies used a simplified description of the heating/cooling properties of Pluto's atmosphere proposed by Yelle \& Lunine (1989), with heating in the methane $3.3 \mu \mathrm{m}$ band and cooling in its $7.6 \mu \mathrm{m}$ band, both occurring in non-local thermodynamic equilibrium (non-LTE) conditions. Lellouch (1994) first suggested that additional cooling due to LTE CO emission rotational lines was important, based on an estimated abundance of $\mathrm{CO}$ in Pluto's atmosphere $\left(10^{-4}-10^{-3}\right)$.

These studies were updated with the much more extensive model of Strobel et al. (1996). Notably these authors improved the treatment of solar heating in the $\mathrm{CH}_{4}$ near-infrared bands by considering the effects of opacity and vibrational $(\mathrm{V}-\mathrm{V}$ and $\mathrm{V}-$ $\mathrm{T}$ ) energy transfer, and showed the need to include heating from the $2.3 \mu \mathrm{m}$ band system in addition to the $3.3 \mu \mathrm{m}$ bands.

As the composition of Pluto's atmosphere, as well as surface (pressure, radius) conditions, were largely unconstrained at that time, Strobel et al. (1996) explored diverse combinations of surface pressure and methane mixing ratios (including nonuniform ones), also including the effect of $\mathrm{CO}$ cooling. In general these models were reasonably successful at explaining large near-surface temperature gradients, though (i) fitting $10-20 \mathrm{~K} \mathrm{~km}^{-1}$ gradients near the surface required pushing the models to their limits, e.g., a $3.6 \% \mathrm{CH}_{4}$ mixing ratio confined to the first scale height near the surface and a $3 \mu \mathrm{bar}$ surface (or tropopause) pressure; and (ii) models tended to overestimate the upper atmosphere temperature $(\sim 130 \mathrm{~K}$ instead of $100 \mathrm{~K})$. A general feature of the Strobel et al. (1996) models was their prediction of a mostly isothermal atmosphere at pressures less than $\sim 2 \mu$ bar, though some models exhibited a moderate ( 0 to $-0.1 \mathrm{~K} \mathrm{~km}^{-1}$ negative gradient at $1-2 \mu \mathrm{bar}$. As the direct detection of $\mathrm{N}_{2}$ in Pluto's atmosphere is still missing, they also considered a CO-dominated atmosphere case (e.g., 97\% CO + $3 \% \mathrm{CH}_{4}$ ). This case led, through enhanced $\mathrm{CO}$ cooling, to much larger negative temperature gradients in the sub-microbar region and an upper atmosphere temperature of about $55 \mathrm{~K}$.

The availability of new, quantitative, observational constraints on the composition $\left(\mathrm{CH}_{4} \sim 0.5 \%, \mathrm{CO} \sim 0.05 \%\right)$ and near-surface structure (surface radius and pressure, tropospheric depth) of Pluto's atmosphere from near-IR observations (Lellouch et al. 2009, 2011) prompted a revival of the Strobel et al. (1996) models (Zalucha et al. 2011a, 2011b; Zhu et al. 2014).

Model updates included new estimates of the vibrational energy transfer based on recent laboratory measurements of collisional relaxation rates (Siddles et al. 1994; Boursier et al. 2003), as well as the introduction of a scheme parameterizing the processes of eddy mixing and convection. With the updated model, Zalucha et al. (2011a) explored the effect of parameter space $\left(\mathrm{CH}_{4}\right.$ and $\mathrm{CO}$ mixing ratios, surface pressure, and radius) allowed by the recent observations, assuming uniform vertical mixing of $\mathrm{CH} 4$ and $\mathrm{CO}$ (which was recently demonstrated to be the case for $\mathrm{CH}_{4}$ in the first $25 \mathrm{~km}$ of the atmosphere; Lellouch et al. 2015a). Radiative-convective calculations were then coupled to a model generating synthetic occultation light curves for direct comparison to observations. The study was extended by Zalucha et al. (2011b) to include a putative troposphere. 

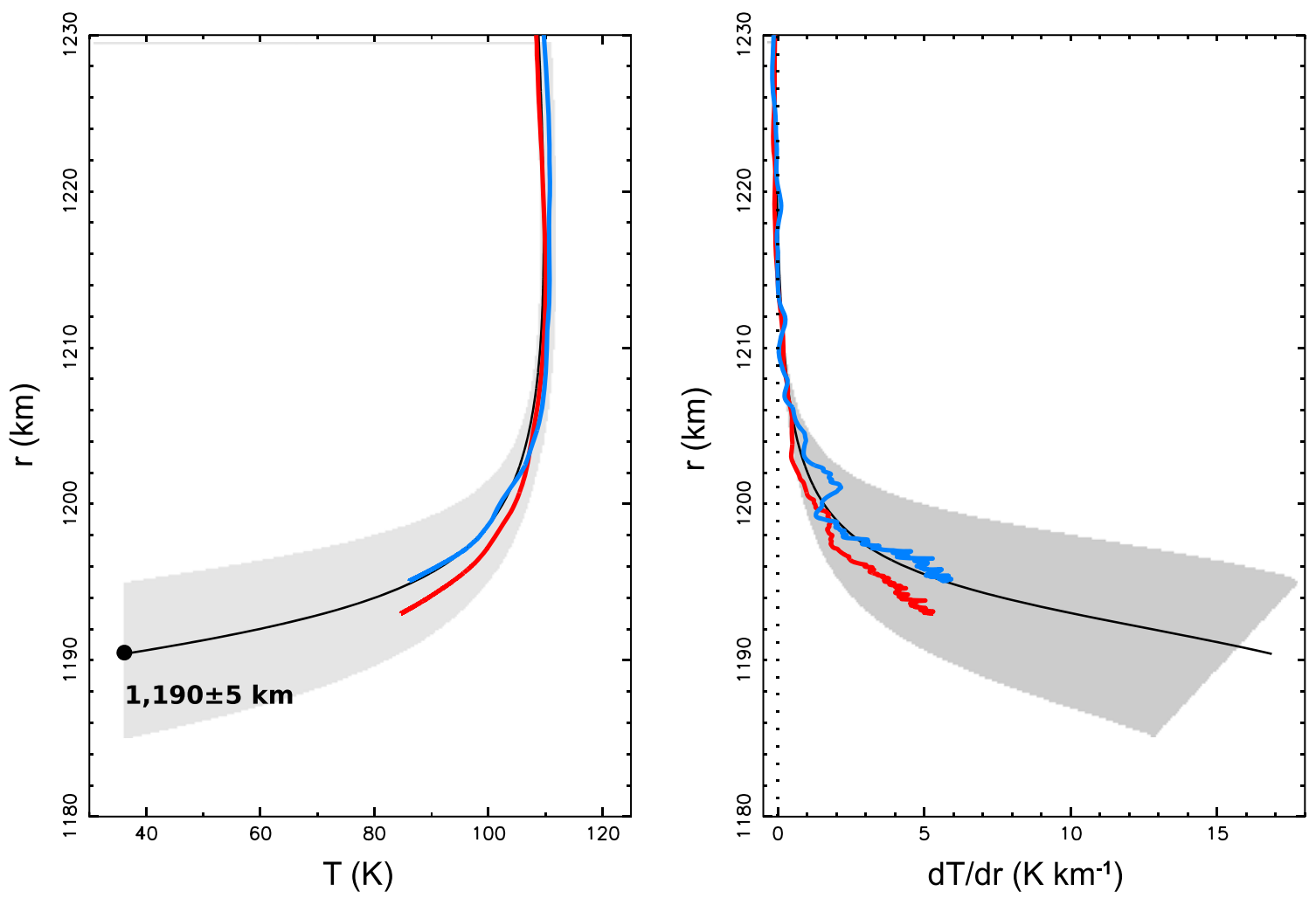

Figure 9. Left: expanded view of the bottom of the temperature profiles shown in Figure 8. The bullet is the intersection with the nitrogen condensation line. The error bar attached to its positions is defined by the radial extension of the shaded uncertainty domain. Right: the corresponding expanded view for the temperature gradient.

In spite of minor changes, the Zalucha et al. (2011a, 2011b) models confirm the essential features of the earlier Strobel et al. (1996) models. The stratopause temperature is still somewhat too high $(120-125 \mathrm{~K})$ near the $1215 \mathrm{~km}$ radius in Zalucha et al. (2011a). These models generally show only weak negative temperature gradients above this level, typically a $\sim 5 \mathrm{~K}$ decrease over a $300 \mathrm{~km}$ range for a $\mathrm{CO}$ mixing ratio of $5 \times 10^{-4}$, or mild $\sim 10 \mathrm{~K}$ decrease due to atmospheric escape (Zhu et al. 2014). This is in disagreement with the profile reported in the present study, which exhibits a typical $30 \mathrm{~K}$ decrease between 1215 and $1390 \mathrm{~km}$ and a gradient of $-0.2 \mathrm{~K} \mathrm{~km}^{-1}$, as discussed earlier. Rather, the profile we derive is remarkably similar to that calculated by Zalucha et al. (2011a; their Figure 8) for the case of 40-times enhanced CO mixing ratio $\left(200 \times 10^{-4}\right)$ This scenario, however, is at odds with the direct measurement of the $\mathrm{CO}$ abundance (Lellouch et al. 2011). This suggests that an additional cooling source is at work.

Through radiation in its intense rotational lines, $\mathrm{HCN}$ is the major cooling agent in Titan's upper atmosphere, where its mixing ratio is typically $2 \times 10^{-4}$ at $1100 \mathrm{~km}$ (Vuitton et al. 2007), and where it equilibrates the solar UV heating rates (Yelle 1991). HCN has not yet been detected in Pluto's atmosphere, but its presence is expected from coupled photochemistry in a $\mathrm{N}_{2}-\mathrm{CH}_{4}$ atmosphere. However, a complete reassessment of the Pluto models is beyond the scope of the present study. Here we only recalculate $\mathrm{CO}$ cooling rates and also examine the case of $\mathrm{HCN}$ cooling. Photochemical models predictions lead to rather diverse mixing ratios of $\mathrm{HCN}\left(10^{-8}\right.$ $10^{-3}$ of $\mathrm{N}_{2}$ (Lara et al. 1997; Summers et al. 1997; Krasnopolsky \& Cruikshank 1999), where the difference largely seems to come from the fact that the more "optimistic" models have not accounted for the fact that under cold $(<100$ $\mathrm{K})$ temperatures, atmospheric condensation of $\mathrm{HCN}$ should occur. Here, we nominally consider cases in which the HCN abundance is limited by the saturation law (Fray \& Schmitt 2009), but we also run a case with uniformly mixed $\mathrm{HCN}$, as supersaturation may be possible in a clear, tenuous atmosphere such as as Pluto's. Cooling rates at radius $r$ are calculated from the following equation (e.g., for $\mathrm{CO}$ ):

$$
R_{\mathrm{CO}}(r)=4 \pi N_{\mathrm{CO}}(r) \int B_{\nu}(T(r)) k_{\nu} E_{2}\left(\tau_{\nu}\right) d \nu
$$

where $N_{\mathrm{CO}}$ is the local CO number density, $T$ is temperature, $k_{\nu}$ and $\tau_{\nu}$ are the absorption coefficients and zenithal opacity at frequency $\nu$, and $E_{2}(\tau)$ is the second exponential integral. The integral runs over the entire millimeter/submillimeter range $\left(0-200 \mathrm{~cm}^{-1}\right)$, and unlike in Strobel et al. (1996), we include all isotopic variants of $\mathrm{CO}$ and $\mathrm{HCN}$, i.e., lines of $\mathrm{CO}, 13 \mathrm{CO}$, $\mathrm{C} 18 \mathrm{O}, \mathrm{HCN}, \mathrm{H} 13 \mathrm{CN}$, and $\mathrm{HC} 15 \mathrm{~N}$ are taken into account when calculating the opacities. Moreover, absorption coefficients are calculated using a Voigt profile, instead of the Doppler approximation. Both aspects lead to a minor but non-negligible increase in the cooling rate at low altitudes. Calculations of the cooling rates are performed for the thermal profile inferred in this work.

Results are shown in Figure 11 for a series of assumed $\mathrm{CO}$ and $\mathrm{HCN}$ profiles. The $\mathrm{CO}$ mixing ratio $q_{\mathrm{CO}}=5 \times 10^{-4}$ curves show the "nominal" CO cooling. Although Zalucha et al. (2011a, 2011b) do not show their cooling rates, our calculation for CO can be compared to Figure 5(a) of Zhu et al. (2014), showing reasonable agreement. Increasing the $q_{\mathrm{CO}}$ by a 


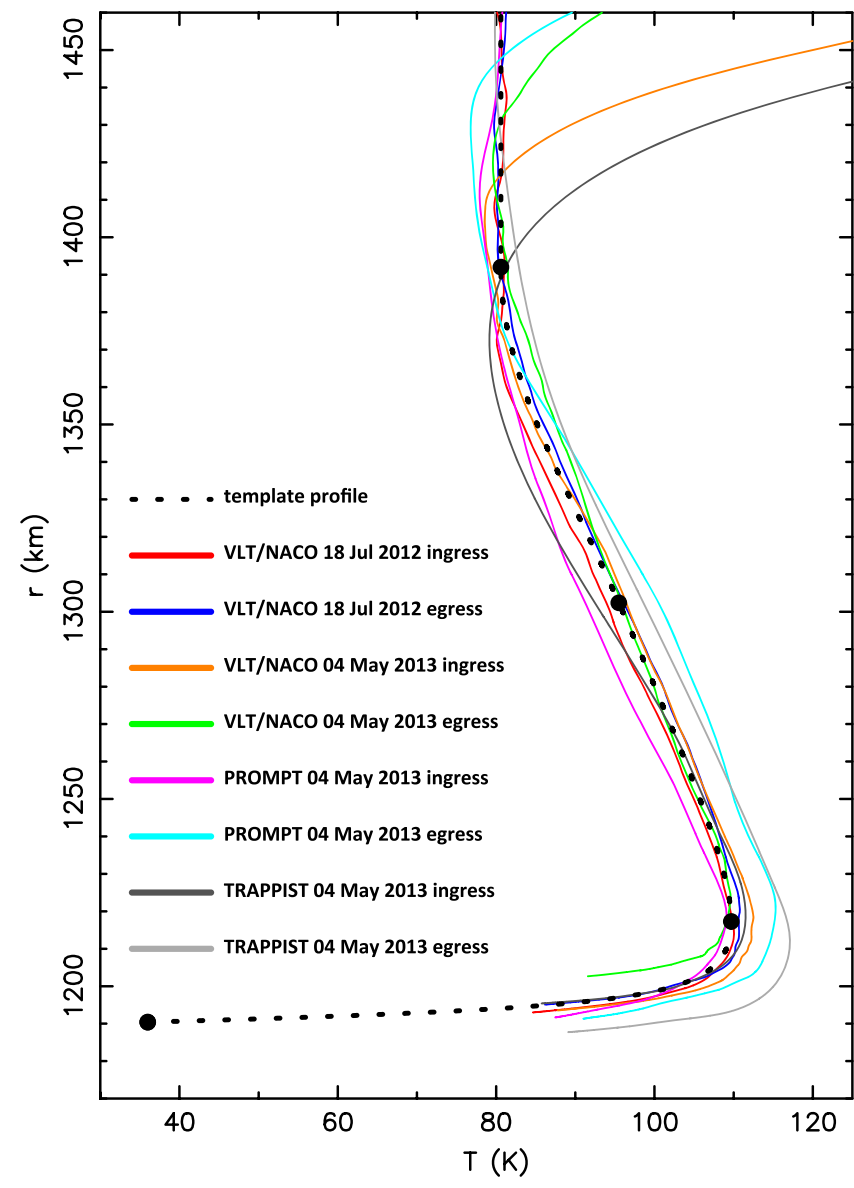

Figure 10. Temperature profiles derived from the inversion of our best occultation light curves obtained on 2012 July 18 and 2013 May 04. The dotted line is our global, best-fitting temperature profile (also shown in Figures 8 and 13).

factor of 40 leads to an increase of the cooling rate, although in much lower proportion due to opacity effects.

Other curves in Figure 11 show the cooling due to HCN for different assumed $\mathrm{HCN}$ mixing ratios in the non-saturated part of the atmosphere. For the temperature profile derived in this work, the lower temperatures above $1270 \mathrm{~km}$ radius, severely restrict the amount of gaseous $\mathrm{HCN}$ if saturation of $\mathrm{HCN}$ is accounted for. In fact, HCN appears to be saturated everywhere in the atmosphere, except possibly over the $1210-1270 \mathrm{~km}$ range, where the condensation law allows $10^{-7}-10^{-6} \mathrm{HCN}$ mixing ratios. There, the $\mathrm{HCN}$ cooling rate may slightly exceed the nominal $\mathrm{CO}$ cooling rate (pink versus red curves in the left panel of Figure 11). However, for the $\mathrm{HCN}$ cooling rates to approach the "enhanced" CO cooling rates necessary to explain our negative mesospheric temperature gradient (i.e., those for $q_{\mathrm{CO}}=200 \times 10^{-4}$, as considered by Zalucha et al. 2011a), one must assume that $\mathrm{HCN}$ is not limited by saturation. Specifically, the blue curve in Figure 11 shows that a uniform $\mathrm{HCN}$ mixing ratio of $\sim 5 \times 10^{-5}$ is required.

Although a full reassessment of the radiative models should be undertaken at this point, we conclude from this exercise that there is no obvious "culprit" for a $-0.2 \mathrm{~K} \mathrm{~km}^{-1}$ temperature gradient above the radius of $\sim 1220 \mathrm{~km}$. According to the calculations of Zalucha et al. (2011a), CO in amounts consistent with the direct observations of Lellouch et al. (2011) provides unsufficient cooling. We show here that $\mathrm{HCN}$ could be an alternative efficient cooling agent, but only if its mixing ration vastly exceeds expectations from the condensation law. Direct measurements/upper limits of $\mathrm{HCN}$ from ALMA and perhaps from the New Horizons UV spectrometer (ALICE) will shed new light on this issue. ${ }^{29}$

\subsection{Alternative Explanations}

Coming back a step, the primary result derived from a stellar occultation light curve is the refractivity profile $\nu(r)$, from which a density profile $n(r)=\nu(r) / K$ is obtained, assuming a given gas composition (Equation (7)).

A first idea is to envisage that hazes are present in the mesosphere. Those hazes would absorb part of the stellar flux, in such a way that a basically isothermal mesosphere is thought to host a negative thermal gradient just because of the clearatmosphere assumption. To test that hypothesis, we have generated synthetic light curves, forcing the mesosphere to be isothermal at $T_{\text {iso }}=95.5 \mathrm{~K}$ above the stratopause (we have also tested other values of $T_{\text {iso }}$ between 85 and $110 \mathrm{~K}$, with the same conclusions). Figure 3 shows the resulting residuals for the NACO 2012 July 18 light-curve (labelled "iso." in that figure). They depart from zero well above the noise level, and we can rule out photometric fluctuations caused by absorbing haze layers, since the residuals have both positive and negative values.

That said, two assumptions may be wrong in Equation (5): (1) the atmosphere may be not composed of pure nitrogen $\mathrm{N}_{2}$, so that the nitrogen molecular mass $\mu$ must be replaced by a new value $\mu^{\prime}$, and (2) hypothetical zonal winds may create a centrifugal acceleration, so that the acceleration of gravity $g$ must be replaced by a term $g^{\prime}$ that includes supplementary terms.

In fact, we can use Equation (5) in a reversed way. More precisely, the refractivity profile $\nu(r)$ is actually an imposed observable (since it is directly derived from the occultation light curve), while we may use a prescribed temperature profile $T^{\prime}(r)$, taken, for instance, from a theoretical model. With this approach in mind, Equation (5) can be rewritten as

$$
\frac{\mu^{\prime} g^{\prime}}{\mu g}=-\frac{k T^{\prime}}{\mu g} \frac{d \log \left(\nu T^{\prime}\right)}{d r} .
$$

To obtain this equation, we have used $\nu(r)=K \cdot n(r)$, where $K$ is the molecular refractivity (Equation (7)). We assume here that the atmospheric composition varies slowly with radius (i.e., $K$ suffers small variations over one scale height), so that we neglect $d K / d r$, and finally provide $d[\log (\nu)] /$ $d r \sim d[\log (n)] / d r$.

Thus, the ratio $\mu^{\prime} g^{\prime} / \mu g$ is the factor by which the molecular mass and/or the acceleration of gravity $g$ must be multiplied in order to retrieve a prescribed temperature profile $T^{\prime}(r)$, given an observed occultation light-curve.

In Figure 12, we consider an example where the prescribed temperature profile $T^{\prime}(r)$ exhibits a decrease of only $10 \mathrm{~K}$ between the stratopause and the mesopause. This is typical of what can be obtained by the combined effects of $\mathrm{CO}$ cooling (Zalucha et al. 2011a) and/or an atmospheric escape (Zhu et al. 2014). The right panel of Figure 12 shows the resulting

\footnotetext{
29 The detection of HCN in Pluto's atmosphere, using ALMA, was announced by Lellouch et al. (2015b) on 2015 July 30.
} 

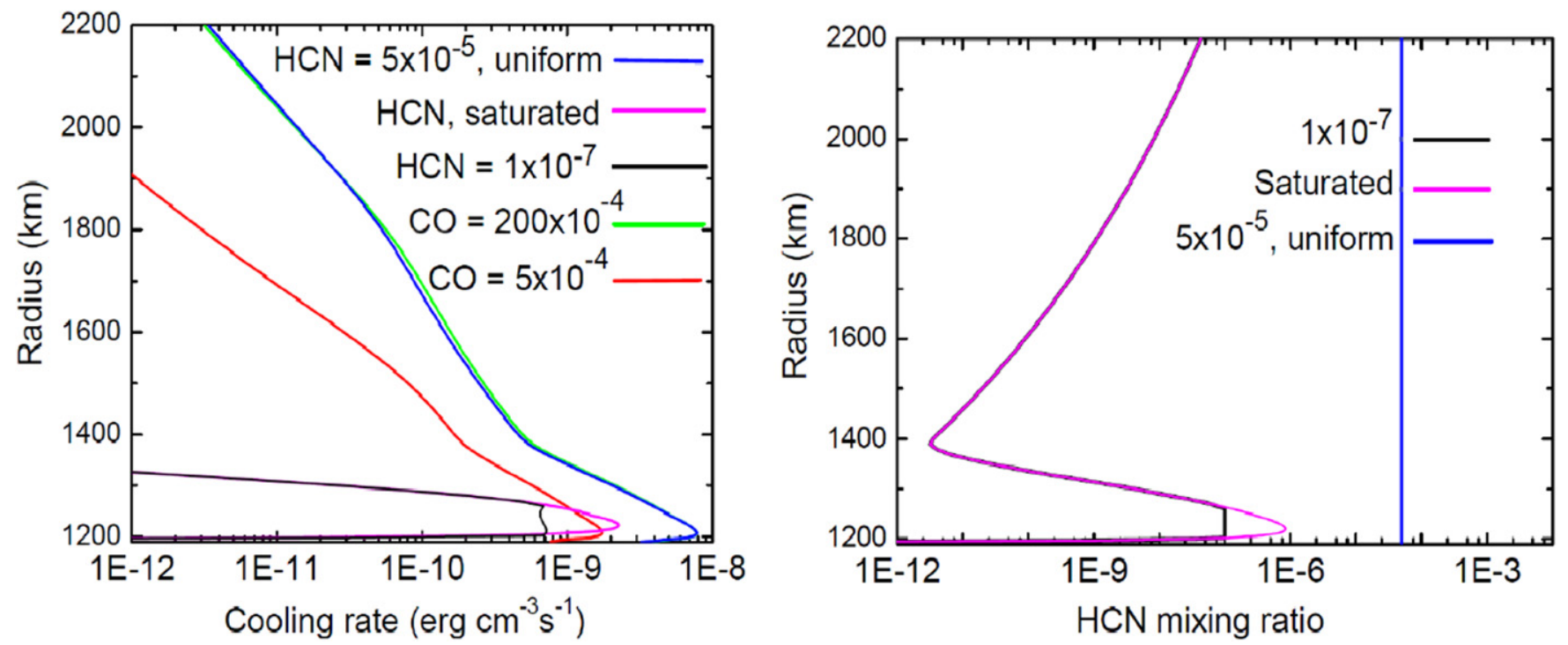

Figure 11. Calculation of cooling rates by CO and HCN. The $y$-axis is the distance from Pluto's center, with the surface position assumed here at $1,184 \mathrm{~km}$. Left panel: cooling rates assuming the thermal profile from this work. Red and green curves: CO cooling rates for $q_{\mathrm{CO}}=5 \times 10^{-4}$ and $200 \times 10^{-4}$, respectively. The other three colored curves show the HCN cooling rate for the corresponding HCN profiles. Right panel: HCN mixing ratios profiles. The black and purple curves make use of the thermal profile from this work. Due to the significantly cold temperatures above $\sim 1300 \mathrm{~km}, \mathrm{HCN}$ is limited by saturation throughout the atmosphere, except in a limited region at $1210-1270 \mathrm{~km}$ for an assumed $q_{\mathrm{HCN}}=10^{-7}$. The blue curve shows the hypothetical case of a uniform (i.e., non-limited by saturation) $5 \times 10^{-5}$ uniform HCN mixing ratio.

profile for $\mu^{\prime} g^{\prime} / \mu g$, restricting ourselves to the mesospheric region.

We first assume here that the atmosphere is composed of pure nitrogen, so that $\mu^{\prime}=\mu$, and the ratio $\mu^{\prime} g^{\prime} / \mu g=g^{\prime} / g$ is only caused by variations of $g^{\prime}$. In the presence of a zonal wind with velocity $v$, the centrifugal acceleration provides $g^{\prime}=g-v^{2} / r$, and from $g=G M / r^{2}$, a zonal wind of:

$$
v=\sqrt{\frac{G M}{r}} \cdot \sqrt{1-\frac{g^{\prime}}{g}} \sim 840 \sqrt{1-\frac{g^{\prime}}{g}} \mathrm{~m} \mathrm{~s}^{-1},
$$

where we have used the value of GM in Table 3 and $r \sim 1250$ $\mathrm{km}$. With the example above, the factor $g^{\prime} / g^{\prime}$ reaches a minimum value of about 0.95 , yielding $v \sim 190 \mathrm{~m} \mathrm{~s}^{-1}$. This is close to supersonic, as the speed of sound for nitrogen $\mathrm{N}_{2}$ at $100 \mathrm{~K}$ is about $200 \mathrm{~m} \mathrm{~s}^{-1}$. In fact, current general circulation models for Pluto predict zonal winds of less than $10 \mathrm{~m} \mathrm{~s}^{-1}$ at the altitudes considered here (Vangvichith 2013; Zalucha \& Michaels 2013). Moreover, we see that above $r \sim 1300 \mathrm{~km}$, the ratio $g^{\prime} / g$ becomes larger than unity with the example considered here, which is impossible from Equation (3). Other prescribed profiles $T^{\prime}(r)$ could be imagined to avoid this problem by displacing the $\mu^{\prime} g^{\prime} / \mu g$ profile to the left in Figure 12 (providing smaller values of $g^{\prime} / g$ ), but this would imply even more unrealistic, high zonal winds.

Considering that $g^{\prime} / g \sim 1$ from the discussion above, the $\mu^{\prime} g^{\prime} / \mu g$ profile would represent variations of the atmospheric molecular weight, $\mu^{\prime} / \mu$. In the example of Figure 12, the molecular weight of the atmosphere has to be inferior to that of molecular nitrogen, $\mu$, to mimic the effect of a negative temperature gradient. This could be caused by the presence of a lighter gas, such as neon, which has a molecular weight of $\mu_{\mathrm{Ne}} \sim 0.72 \mu$. That species has a relatively large solar abundance $\left(N_{e} / N \sim 1.5\right)$ and is not condensed at Pluto's atmosphere's temperatures. The minimum value $\mu^{\prime} / \mu=0.95$ near $1,230 \mathrm{~km}$ (Figure 12) would then require a local neon abundance of about $82 \%$. However, and as before, the ratio $\mu^{\prime} / \mu$ would be larger than unity above $1300 \mathrm{~km}$, requiring that another heavier gas (e.g., argon) takes over above $1300 \mathrm{~km}$ and drives the molecular mass upward. Such a model is clearly unrealistic though, because mass separation would result in a depletion, not enrichment, of the heavier species in the upper atmosphere.

\section{UPPER ATMOSPHERE}

Above $r \sim 1400 \mathrm{~km}$, the results of Figure 8 show that a change of thermal gradient may occur, with a more isothermal upper branch just above the mesosphere. However, the lack of independent constraints on the temperature at that level prevents an unambiguous choice for a particular solution for $T(r)$. In addition, the rapidly increasing contribution of the noise makes any estimation of the thermal gradient above $1450 \mathrm{~km}$ (Figure 8, panel (d)) impossible. That said, the lack of obvious mechanisms to drastically warm up or cool down the atmosphere just above $1400 \mathrm{~km}$ suggests (but by no means proves) an isothermal branch between 1400 and $1450 \mathrm{~km}$. Under this hypothesis, we estimate a $1 \sigma$ uncertainty domain of $81 \pm 6 \mathrm{~K}$ for the temperature of this isothermal branch. (This interval corresponds to an increase of $\Delta \chi^{2}=+1$ of the $\chi^{2}$ function with respect to the best, minimum value $\chi_{\min }^{2}$ ).

\section{DISCUSSION AND CONCLUSIONS}

We have analyzed some of the best light curves ever obtained during stellar occultations by Pluto. A combination of well-sampled occultation chords (Figure 2) and high S/N data (Figures 3, 4) has allowed us to constrain the density, temperature, and thermal gradient profiles of Pluto's atmosphere between radii $r \sim 1190 \mathrm{~km}$ (pressure $p \sim 11 \mu$ bar) and $r \sim 1450 \mathrm{~km}$ (pressure $p \sim 0.1 \mu \mathrm{bar}$ ). Our main results are listed below. 

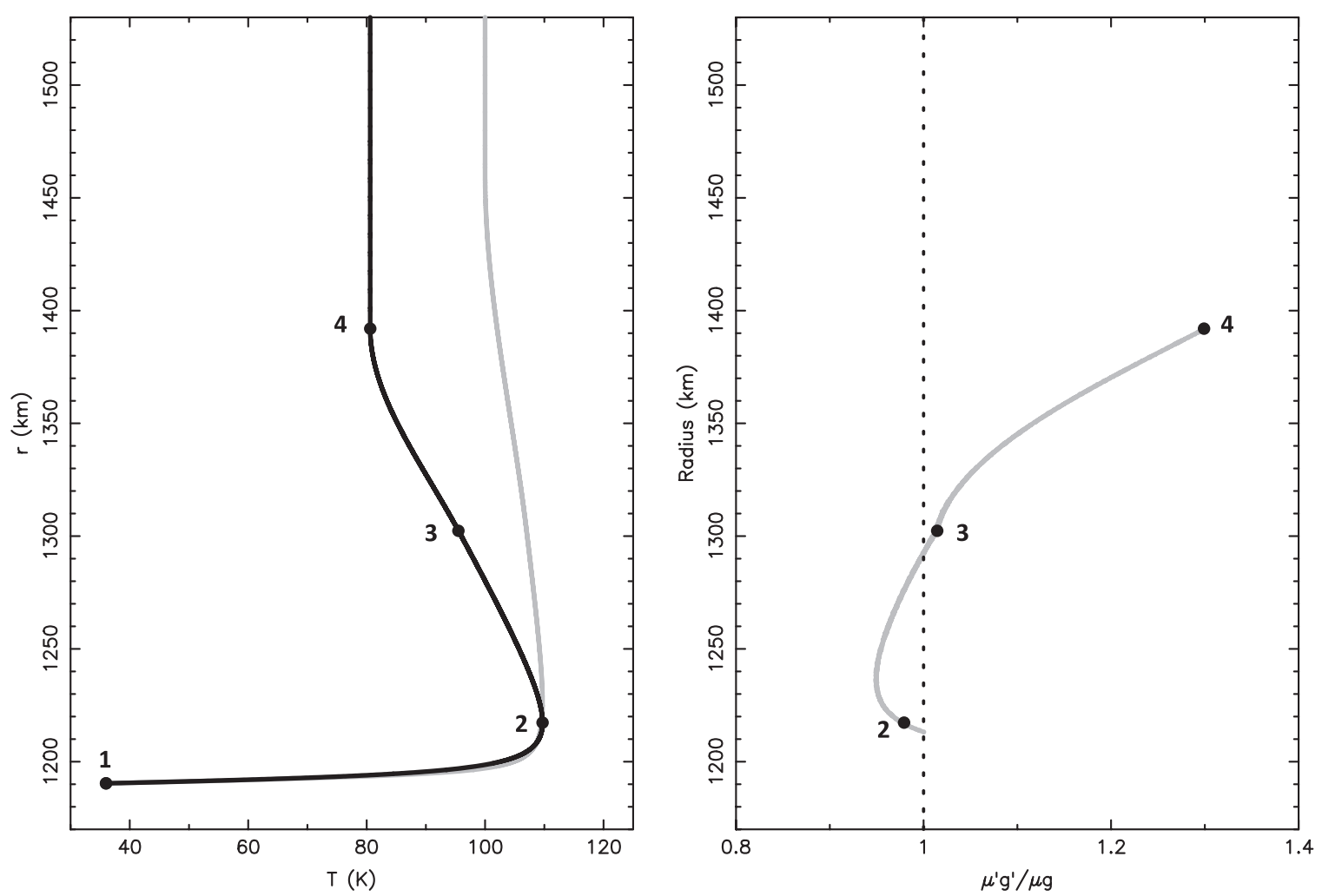

Figure 12. Left panel: solid line: our best temperature profile (see Figure 13). Gray line: an example of a prescribed profile with a milder mesospheric thermal gradient, here a $10 \mathrm{~K}$ drop between the stratopause and the mesopause. Right panel: the ratio $\mu^{\prime} g^{\prime} / \mu g$, as defined by Equation (2), corresponding to the gray, prescribed profile of the left panel. The points numbered 2-4 respectively correspond to the stratopause, the inflexion point, and the mesopause (see also Figure 13 and the text for details).
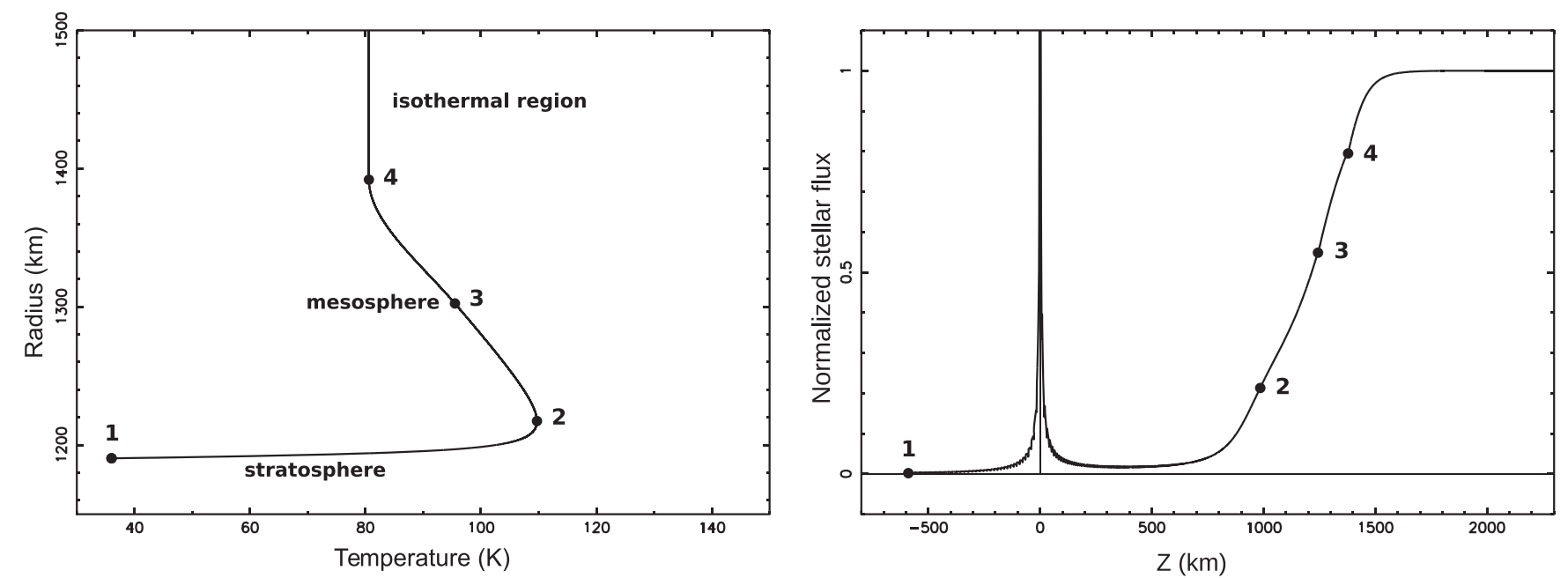

Figure 13. Left: the temperature profile $T(r)$ that best fits our 2012 and 2013 light curves (see also Figures 3 and 4). The parameters used to generate this profile are given in Table 4. Total thickness of the atmosphere: $1100 \mathrm{~km}$; vertical sampling of the model: $0.03 \mathrm{~km}$. Right: the corresponding synthetic flux in the shadow plane for the 2012 July 18 occultation. Here, $z$ is the distance to the shadow center, with the four points corresponding to those of the left panel.

Global Pluto's atmospheric model. We find that a unique thermal model can satisfactorily fit 12 light curves observed in 2012 and 2013 (Figures 3 and 4), assuming a spherically symmetric and clear (no haze) atmosphere. The parameters defining our best model are listed in Table 4 (see also Figure 13), and the various resulting profiles (density, temperature, and thermal gradient) are displayed in Figures 810. The absolute vertical scale of our global model has an internal accuracy of about $\pm 1 \mathrm{~km}$ (Table 4). However, this error is amplified to $\pm 5 \mathrm{~km}$ at the bottom of the profiles (Figure 9), because of the uncertainty on the residual stellar flux (Figure 7) in the central part of the occultation observed by NACO on 2012 July 18.

We quantify in this work the propagation of the photometric noise into the density, temperature, and thermal gradient profiles (Equation (14) and Figure 8). The key parameter that governs the noise propagation is the radius $r_{0}$ in the atmosphere, at which the stellar drop caused by differential 
refraction is equal to the flux standard deviation. The radius $r_{0}$ can be estimated from Equation (13), which includes all of the quantities at work in a stellar occultation: photometric noise, molecular refractivity, atmospheric scale height and radius, and distance to the body. For the NACO light curve, we find $r_{0}=$ $1565 \mathrm{~km}$, corresponding to a pressure level of about 14 nbar.

Although a satisfactory fit to all the data used here is provided by a unique model, there are two slight, but significant departures from this global model. They are now discussed in turn.

Pressure increase between 2012 and 2013. In the frame of our model (i.e., assuming a constant temperature profile), we detect a significant $6 \%$ pressure increase (at the $6 \sigma$ level) during the $\sim 9.5$ months separating the two events under study. This means that Pluto's atmosphere was still expanding at that time, confirming the work of Olkin et al. (2015), which compiles and analyzes pressure measurements between 1988 and 2013.

Ingress/egress asymmetry of lower temperature profiles. Figure 7 shows that the stellar flux decreased from $2.3 \%$ to $1.8 \%$ of its unocculted value during the central part of the 2012 July 18 occultation, as observed by NACO from Paranal. This corresponds to the primary stellar image first scanning the summer, permanently lit Pluto northern hemisphere, and then the winter low-insolation southern hemisphere (Figure 2). This confirms a similar trend pointed out by Sicardy et al. (2003) during another high $\mathrm{S} / \mathrm{N}$ stellar occultation recorded in 2002 August. These authors interpreted this result as a surface boundary layer effect, where the lowermost scale height adjusts itself to the surface temperature variegations, which might explain the behavior displayed in Figure 9.

Another interpretation of this trend is the gradual entrance of the primary stellar image into an absorbing haze layer near Pluto's evening limb, a hypothesis that can be tested during the New Horizons flyby in 2015 July.

Pluto's radius and density. The extrapolation of our temperature profiles to the nitrogen saturation line implies that nitrogen may condense at Pluto's radius of $R_{\mathrm{P}}=1190 \pm 5 \mathrm{~km}$. However, the few kilometers above Pluto's surface remain "terra incognita" as far as stellar occultations are concerned. In particular, the temperature gradients shown in Figure 9 may deviate from the simple extrapolation used here, especially if haze layers affect the retrieved temperature profiles. Although difficult to envisage because of the strong caustics that they cause, a troposphere below $1190 \pm 5 \mathrm{~km}$ cannot be excluded. Combining high-resolution spectroscopic observations of gaseous methane, combined with constraints from an occultation observed in 2002, Lellouch et al. (2009) conclude that the troposphere depth cannot exceed about $17 \mathrm{~km}$. Consequently (and assuming that the temperature of the deep atmosphere did not change significantly since 2002), our observations constrain Pluto's radius to lie in the range $1168-1195 \mathrm{~km}$. More recently, combining constraints from spectra and a preliminary analysis of the occultation data presented in this work, Lellouch et al. (2015a) concluded that Pluto should have a radius between 1180 and $1188 \mathrm{~km}$, some $2-8 \mathrm{~km}$ below the condensation radius of $1190 \mathrm{~km}$ that we derive above.

From Pluto's mass of $M_{\mathrm{P}}=1.304 \pm 0.006 \times 10^{22} \mathrm{~kg}$ (Tholen et al. 2008), we derive a density $\rho_{\mathrm{P}}=$ $(1.802 \pm 0.007)\left(R_{\mathrm{P}} / 1200 \mathrm{~km}\right)^{-3} \mathrm{~g} \mathrm{~cm}^{-3}$. Our estimation $R_{\mathrm{P}}=$ $1190 \pm 5 \mathrm{~km}$ thus implies $\rho_{\mathrm{P}}=1.85 \pm 0.02 \mathrm{~g} \mathrm{~cm}^{-3}$ in the absence of a troposphere, and a range $\rho_{\mathrm{P}}=1.83-1.95 \mathrm{~g} \mathrm{~cm}^{-3}$ if a troposphere is allowed. This is larger, but not by much, than Charon's density, $\rho_{\mathrm{C}}=1.63 \pm 0.05 \mathrm{~g} \mathrm{~cm}^{-3}$ (Ibid.).

The mesospheric negative thermal gradient. Pluto's stratopause occurs near $1215 \mathrm{~km}$ (pressure $p=6.0 \mu \mathrm{bar}$ ), with a maximum temperature of $110 \pm 1 \mathrm{~K}$, where the error bar applies to the best inverted profile (NACO 2012 July 18), and stems from the uncertainty on the Pluto + Charon flux contribution (Figure 8).

Above the stratopause, and up to about $1390 \mathrm{~km}$, our best 2012 and 2013 occultation light curves yield inverted temperature profiles with a negative thermal gradient close to $-0.2 \mathrm{~K} \mathrm{~km}^{-1}$, which amounts to a total decrease of $30 \mathrm{~K}$ for the temperature between 1215 and $1390 \mathrm{~km}$ (Figures 8, 10).

Explaining this negative gradient by $\mathrm{CO}$ cooling requires a mixing ratio $\left(200 \times 10^{-4}\right)$ that is too high by a factor of 40 compared to current measurements (Lellouch et al. 2011). Cooling by $\mathrm{HCN}$ is also discussed in this paper. It appears to be a possible alternative solution, but only if it remains largely supersaturated in the mesosphere.

Changing the temperature boundary condition may suppress the negative gradient, but at the expense of creating a warm, unexplained thermal profile above $1350 \mathrm{~km}$. We have investigated more exotic solutions, like zonal winds or compositional variations that would "unbend" the retrieved temperature profiles, allowing a more isothemal mesosphere. However, no realistic models could be built upon those alternative assumptions. Again, the New Horizons flyby will provide constraints on the temperature boundary conditions and atmospheric composition that will be used to discriminate between the various solutions described here.

We acknowledge support from the French grants "Beyond Neptune" ANR-08-BLAN-0177 and "Beyond Neptune II" ANR-11-IS56-0002.

A. Dias-Oliveira is thankful for the support of the following grants: CAPES (BEX 9110/12-7) FAPERJ/PAPDRJ (E-45/ 2013). R. Vieira-Martins acknowledges the following grants: CNPq-306885/2013, CAPES/Cofecub-2506/2015, FAPERJ/ PAPDRJ-45/2013. TRAPPIST is a project funded by the Belgian Fund for Scientific Research (F.R.S.-FNRS) under grant FRFC 2.5.594.09.F, with the participation of the Swiss National Science Foundation. J. L. Ortiz and N. Morales acknowledge funding from Proyecto de Excelencia de la Junta de Andalucía, J.A. 2012-FQM1776 and from FEDER funds. M. Gillon and E. Jehin are F.R.S.-FNRS Research Associates. C. Opitom acknowledges the support of the F.R.S.-FNRS for her $\mathrm{PhD}$ thesis. R. Leiva is supported by the CONICYT PCHA/Doctorado Nacional scholarship. M. Assafin thanks the CNPq (Grants 473002/2013-2 and 308721/2011-0) and FAPERJ (Grant E-26/111.488/2013). J.I.B. Camargo acknowledges CNPq for a PQ2 fellowship (process number 308489/2013-6). A.R. Gomes-Júnior thanks CAPES. We also thank Caisey Harlingten for the use of his 20 inch telescope in San Pedro de Atacama.

\section{APPENDIX A SYNTHETIC LIGHT CURVES}

\section{A.1. Parameterized Temperature Profile}

We define a parametric model for Pluto's atmosphere temperature profile, $T(r)$, where $r$ is the radius, i.e., the distance to Pluto's center. The model must be detailed enough to capture 
the main features revealed by the inversions (Figures 10 and 13), but still simple enough to allow an easy and meaningful control of $T(r)$ and an assessment of the error bars associated with each parameter. The features we want to describe are: (i) a thin stratosphere just above the surface, with a strong increase of temperature with altitude, (ii) an "elbow" where the temperature reaches its maximum, marking the stratopause, (iii) an intermediate region with a mild negative gradient, and finally (iv), an isothermal upper branch.

These features define three regions, bounded by four points $1, \ldots 4$ at prescribed radii $r_{1}, \ldots r_{4}$, see Figure 13 . More precisely, the profile $T(r)$ is generated as follows:

$$
\begin{cases}c 1 \cdot r+c 2 \cdot T(r) & \text { for } \quad r_{1} \leqslant r \leqslant r_{3} \\ +c 3 \cdot r T(r)+c 4 \cdot r^{2} & \text { (hyperbolic branch) } \\ +c 5 \cdot T^{2}(r)=1 & \\ T(r)=c 6+c 7 \cdot r & \text { for } r_{3} \leqslant r \leqslant r_{4} \\ +c 8 \cdot r^{2}+c 9 \cdot r^{3} & \text { (polynomial branch) } \\ T(r)=T_{\text {iso }} & \text { for } \quad r_{4} \leqslant r \text { (straight line). }\end{cases}
$$

Note that $r_{2}$ does not appear in the equations above, and is defined as the radius where the temperature reaches its maximum (Figure 13). The functional forms chosen here (hyperbolic, polynomial, and straight lines) are not based on physical grounds, but rather, are empirical and simple formulae that satisfactorily fit the observed profiles (Figure 8).

The parameters $c 1, \ldots c 9$ are determined to ensure that $T(r)$ is continuous both in temperature and its derivative, $d T / d r$, at points 1,3 , and 4 . Those conditions provide algebraic systems that are solved by a classical Gauss-Jordan method (Press et al. 1992).

In practice: (1) we fix the temperature $T_{1}=T\left(r_{1}\right)$ at the bottom of the profile, together with its gradient $(d T / d r)_{1}$. (2) We fix the value of the maximum of temperature $T_{2}=T\left(r_{2}\right)$ at $r_{2}$ and the temperature $T_{3}=T\left(r_{3}\right)$ at inflexion point 3 . We thus have three boundary conditions for $T: T_{1}, T_{2}, T_{3}$ at $r_{1}, r_{2}$, and $r_{3}$, respectively, and two boundary conditions for $d T / d r$ : $(d T / d r)\left(r_{1}\right)=(d T / d r)_{1}$ and $(d T / d r)\left(r_{2}\right)=0$, which fixes the five coefficients $c 1, \ldots c 5$. Note in passing that the values of $c 1, \ldots c 5$ then impose the temperature gradient $(d T / d r)_{3}$ at $r_{3}$; (3) We fix the temperature $T_{\text {iso }}$ at $r_{4}$, the point where the isothermal branch is reached. This provides two boundary conditions in $T: T_{3}$ and $T_{\text {iso }}$ at $r_{3}$ and $r_{4}$, respectively, plus two boundary conditions for $d T / d r:(d T / d r)_{3}$ at $r_{3}$ and $(d T / d r)\left(r_{4}\right)=0$, thus fixing the remaining four coefficients $c 6, \ldots c 9$.

The locations of points 1-4 in the space $(T, r)$ are chosen to best fit the observed profiles (see the main text for details). Once $T(r)$ is defined, the gas number density profile $n(r)$ is obtained by integrating the first order differential equation:

$$
\frac{1}{n} \cdot \frac{d n}{d r}=-\left[\frac{\mu g(r)}{k T}+\frac{1}{T} \cdot \frac{d T}{d r}\right],
$$

derived from the equation of state for an ideal gas, and the hydrostatic equation. Here,

$$
g(r)=\frac{G M}{r^{2}}
$$

is the acceleration of gravity, assuming a spherical, homogeneous planet. The values of $\mu$ (Table 4) correspond to molecular nitrogen, assumed to be the unique gas present in the atmosphere. Also listed in Table 4 are the Boltzmann constant $k$ and Pluto's mass parameter GM.

A boundary condition is required to integrate Equation (5), e.g., the pressure $p_{1275}$ at $r=1275 \mathrm{~km}$, which fixes the needed boundary condition $n_{1275}=P_{1275} / k T_{1275}$. Finally, the refractivity $\nu(r)$ of the gas (index of refraction minus unity) is given by

$$
\nu(r)=K \cdot n(r),
$$

where the molecular refracticity is given in Table 3, assuming again pure molecular nitrogen. Once $\nu(r)$ is obtained, we can derive the vertical refractivity gradient $d \nu / d r$ that is used in the ray-tracing code, see below.

The inversions proceed the other way around: the light curves provide $d \nu / d r(r)$ through an abelian integral (Vapillon et al. 1973), then $\nu(r)$, from which $n(r)$ is derived (Equation (7)), followed by the temperature profile, once a boundary condition is given for $T$ (Equation (5)).

\section{A.2. Ray-tracing}

For small values of $\nu$ (as it is the case here) and under a spherical symmetry assumption, a stellar ray is deviated by $d \omega=(\partial \nu / \partial r) \cdot d s$ (Snell-Descartes law) as it moves along an elementary path $d s$. In principle, a ray-tracing code should account for the curvature of the stellar ray as it is refracted in the atmosphere. In practice, however, it is enough to assume that the ray has a rectilinear trajectory in the entire atmosphere. In fact, the maximum total deviation $\omega$ suffered by the ray is very small for ground-based occultations, more precisely of the order of Pluto's apparent angular radius, $\sim 0.05 \mathrm{arcsec}$, so that $\omega<\sim 3 \times 10^{-7}$ rad. Most of that deviation occurs in the deepest scale height $H$ traversed at radius $r$, which represents a traveled length of $l \sim \sqrt{2 \pi r H}$ (Baum \& Code 1953). Taking typical values of $r \sim 1200 \mathrm{~km}$ and $H<\sim 50 \mathrm{~km}$, we get $l<\sim 600 \mathrm{~km}$, i.e., a deviation inside the atmosphere of $\sim \omega \cdot l<0.2 \mathrm{~m}$, which is negligible compared to the scales probed by ground-based stellar occultations.

The numerical integration of Equation (5), using a second order scheme, provides $n\left(r_{i}\right)$ at discreet layers of radii $r_{i}$, from which the refractivity $\nu_{i}$ and its gradient $(d \nu / d r)\left(r_{i}\right)$ are calculated. The total deviation along the straight line $s$ is then:

$$
\omega=\sum_{i} \Delta \omega_{i}=\sum_{i}(d \nu / d r)\left(r_{i}\right) \cdot \Delta s_{i},
$$

where $\Delta s_{i}$ is the path along $s$ traveled inside the layer $i$. Then, for a closest approach $r$ of a ray to Pluto's center, the corresponding distance $z$ to the shadow center upon arrival on Earth is

$$
z=r+\omega(r) \cdot D,
$$

where $D$ is Pluto's geocentric distance. The observed stellar flux is then

$$
\Phi(z)=f \frac{d r}{d z}=\frac{1}{1+D d \omega / d r},
$$

where $f=r / z$ is the focusing factor due to the (assumed circular) limb curvature (see Sicardy et al. 1999).

The thickness $\Delta r_{i}$ of the individual refracting layers has been adjusted to $30 \mathrm{~m}$ to minimize numerical noise, while keeping computing times reasonably low. Similarly, the sampling for $r$ (the closest distance of the rays to Pluto's center) has been 
adjusted so that adjacent rays arrive at separation $\Delta z \approx 1 \mathrm{~km}$ in the shadow plane.

Once the table $(r, z, \Phi(z))$ has been completed, the synthetic flux at a given site and given moment (corresponding to a distance $z_{\text {obs }}$ of the observer to the shadow center) is calculated by interpolation. If several stellar images are present, all of the fluxes are summed. In the particular case of a spherically symmetric atmosphere, and for a given distance $z_{\mathrm{obs}}$, there is a primary image corresponding to $z=z_{\mathrm{obs}}$, and a secondary image corresponding to $z=-z_{\text {obs }}$.

The lowest radius $r_{1}$ considered in the model $(1190.4 \mathrm{~km}$, see Table 4 and Figure 13) is adjusted so that the corresponding flux received in the shadow plane is $\sim 10^{-3}$ of the unocculted stellar flux, negligible compared to the noise level of the best light curves. The upper limit for the atmosphere has been fixed to a radius of about $2300 \mathrm{~km}$. This corresponds to a pressure level of about $0.05 \mathrm{nbar}$, at which point the stellar drop is several orders of magnitudes less than the noise in our best light curves.

The profile that best fits our light curves is shown in Figure 13 . The trajectories of the primary and secondary stellar images as seen from VLT on 2012 July 18 are displayed in Figure 2.

The best fits to the observed light curves are shown in Figures 3 and 4 . Their quality is assessed through the so-called $\chi^{2}$ per degree of freedom:

$$
\chi_{\mathrm{dof}}^{2}=\frac{\chi^{2}}{N-M}=\frac{1}{N-M} \sum_{i=1}^{N}\left(\frac{\Phi_{\mathrm{obs}, \mathrm{i}}-\Phi_{\mathrm{syn}, \mathrm{i}}}{\sigma_{i}}\right)^{2},
$$

where $\Phi_{\mathrm{obs}, \mathrm{i}}\left(\right.$ resp. $\Phi_{\mathrm{syn}, \mathrm{i}}$ ) is the observed (resp. synthetic) stellar flux of the $i^{\text {th }}$ data point, $\sigma_{i}$ is its associated standard deviation, where the summation is extended to the $N$ data points from all the light curves used in the fit, and $M$ is the number of free parameters of the model. As we have nine coefficients $c_{1}, \ldots c_{9}$ to define $T(r)$ (Equation (4)), a boundary condition $p_{1275}$ and two quantities to define Pluto's center, $M=12$.

\section{APPENDIX B NOISE PROPAGATION}

\section{B.1. Photometric Noise}

Here we estimate the effect of photometric noise in an occultation light curve on the retrieved density, temperature, and temperature gradient profiles. We denote $\delta$ the fluctuation of a given quantity, and $\sigma=\sqrt{\delta^{2}}$ is its standard deviation, where the bar denotes average values. For estimation purposes, it is enough to assume here (but not in the ray-tracing or inversion procedures) that the atmosphere has locally a constant density scale height $H$ that is small compared to the planet radius. The stellar flux is then given by the Baum and Code (BC) equation (Baum \& Code 1953):

$$
\frac{1}{\Phi}-2+\log \left(\frac{1}{\Phi}-1\right)=-\frac{z-z_{1 / 2}}{H},
$$

where $z_{1 / 2}$ is the position in the shadow plane at which $\Phi=1 / 2$ (half-light level).

We focus on the top of the profiles, corresponding to $\Phi \sim 1$, so that Equation (12) becomes $\Phi(z) \sim 1-\exp$ $\left[-\left(z-z_{1 / 2}\right) / H\right]$. Moreover, for $\Phi \sim 1$, the stellar ray deviation $\omega$ is small, and we can equate $r$ and $z$ (see Equations (9) and (10)), where $f \sim 1$. In the $\mathrm{BC}$ approximation, we have $\omega \sim-\nu \sqrt{2 \pi r / H}$, where $\nu$ is the refractivity at $r$. As the atmosphere density profile is basically exponential, $d \omega / d r \sim-\omega / H=\nu \sqrt{2 \pi r / H^{3}}$, so that Equation (10) can be used to estimate the expected refractivity corresponding to a stellar flux $\Phi$, namely $\nu \sim(1-\Phi) \sqrt{H^{3} / 2 \pi r D^{2}}$.

We denote $\nu_{0}$ and $r_{0}$ the refractivity and corresponding radius where the stellar drop is equal to the standard deviation of the flux, $\sigma_{\Phi}$, i.e.,

$$
\nu_{0} \sim \sigma_{\Phi} \sqrt{\frac{H^{3}}{2 \pi r D^{2}}} .
$$

Thus, $r_{0}$ is the radius where the stellar drop starts to be barely significant, given the photometric noise. At the upper part of the profiles, we have $H \sim 60 \mathrm{~km}$. The 2012 July 18 NACO light curve has a photometric standard deviation of $\sigma_{\Phi}=0.011$. Using the value of $D$ given in Table 3, we obtain $\nu_{0} \sim$ $1.3 \times 10^{-11}$. Assuming a pure $\mathrm{N}_{2}$ atmosphere, we obtained the corresponding molecular density $n_{0}=\nu_{0} / K \sim 6 \times 10^{12}$ $\mathrm{cm}^{-3}$, which is reached at radius

$$
r_{0} \sim 1565 \mathrm{~km} \text {. }
$$

For $\Phi \sim 1$ (and $f \sim 1$ ), and using the results above, Equation (10) provides $\Phi \sim 1-D d \omega / d r=1+\sqrt{2 \pi r D^{2} / H}$ $(d \nu / d r)$. For a noise-free light curve, we expect $\Phi=1-\sigma_{\Phi} \exp \left[-\left(r-r_{0}\right) / H\right]$. In reality, $\Phi$ is affected by fluctuations $\delta_{\Phi}$, so the retrieved refractivity gradient is in fact: $d \nu / d r=\left(\nu_{0} / H\right) \cdot\left[-\exp \left[-\left(r-r_{0}\right) / H\right]+\delta_{\Phi} / \sigma\right]$. Consequently, the standard deviation associated with each point of the $(d \nu / d r)(r)$ profile (and restricting ourselves to the top of the profile) is:

$$
\sigma_{d \nu / d r} \sim \frac{\nu_{0}}{H} .
$$

The profile $(d \nu / d r)(r)$ is the primary result derived from the light curve, and from which all the other profiles are deduced. Once $d \nu / d r$ is known, we have to estimate $\nu(r)=\nu_{1}+\int_{r_{1}}^{r}(d \nu / d r) d r$, where $\left(r_{1}, \nu_{1}=\nu\left(r_{1}\right)\right) \quad$ is a boundary condition. The integration is performed numerically by taking $\nu(r)=\nu\left(r_{1}\right)+\sum_{i=1}^{N}(d \nu / d r)\left(r_{i}\right) \cdot \Delta r$, where $\Delta r$ is the spatial sampling of the data (i.e., $\Delta r=$ the star velocity perpendicular to the limb multiplied by the exposure time). Thus, $r_{i}=r_{1}+(i-1) \Delta r$ and $N=\left|r-r_{1}\right| / \Delta r$. Adding the variances associated with individual $(d \nu / d r)\left(r_{i}\right)$ 's, we obtain:

$$
\sigma_{\nu} \sim \sqrt{\frac{\left|r-r_{1}\right|}{H} \frac{\Delta r}{H}} \nu_{0} \sim \sqrt{\frac{\Delta r}{H}} \nu_{0},
$$

where the second approximation stems from the fact that $r_{1}$ is chosen close to $r_{0}$ and which we are considering here the few top scale heights of the profiles, so that $\left|r-r_{1}\right| / H \sim \mathcal{O}(1)$. Note that $\sigma_{\nu}=0$ for $r=r_{1}$. This is because $\left(r_{1}, \nu_{1}\right)$ is an arbitrary boundary condition, and as such has no associated error bars.

From $n=\nu / K$, we obtain the standard deviation associated with the density gradient and the density itself: $\sigma_{d n / d r}=\sigma_{d \nu / d r} / K$ and $\sigma_{n}=\sigma_{\nu} / K$. Moreover, from Equation (5), and assuming an isothermal upper atmosphere, we obtain $\delta_{d T / d r}=-(T / n) \delta_{d n / d r}$, so that $\sigma_{d T / d r}=(T / n) \sigma_{d n / d r}$. Finally, the temperature profile is obtained from the numerical integration of $T(r)=T_{2}+\int_{r_{2}}^{r}(d T / d r) d r, \quad$ where 
$\left(r_{2}, T_{2}=T\left(r_{2}\right)\right)$ is an arbitrary boundary condition. Using the same line of reasoning as for $n(r)$, we obtain $\sigma_{T}$ by adding the variances $\sigma_{d T / d r}^{2}$ of all the points $i=1 \ldots N$ involved in the integration, where now $N=\left|r-r_{2}\right| / \Delta r$. Combining the results above, we obtain the following standard deviations for $n(r), T(r)$ and $(d T / d r)(r)$ :

$$
\begin{aligned}
\sigma_{n} & \sim \sqrt{\frac{\Delta r}{H}} n_{0}, \\
\sigma_{T} & \sim T \sqrt{\frac{\Delta r}{2 H}} \sqrt{\left|e^{2\left(r-r_{2}\right) / H}-1\right|} e^{\left(r_{2}-r_{0}\right) / H}, \\
\sigma_{d T / d r} & \sim \frac{T}{H} e^{\left(r-r_{0}\right) / H} .
\end{aligned}
$$

Figure 8 shows the $\pm 1 \sigma$ envelopes at the upper parts of the various profiles. We take here $r_{2}=1390 \mathrm{~km}$, the radius at which we fix a prescribed temperature $T_{2} \sim 81 \mathrm{~K}$. Note again that $\sigma_{T}=0$ at $r=r_{2}$, as $\left(r_{2}, T_{2}\right)$ is an arbitrary boundary condition. Finally, the envelopes $\pm 1 \sigma$ are plotted only down to the half-light level $(r \sim 1290 \mathrm{~km})$, as the estimations made here apply only for the upper part of the light curve. In any case, below that level, the uncertainties in the profiles are dominated by the uncertainty on the background Pluto + Charon contribution (see below).

\section{B.2. Effect of the Pluto and Charon Flux Contributions}

The stellar flux reaches its minimum value in the shadow at typically $z_{\min } \sim\left(z_{1 / 2}\right) / 2$, i.e., halfway between the half-light level and the shadow center, where the central flash occurs (Figure 13). At the minimum, we have from Equation (12): $\Phi_{\min } \sim H /\left(z_{1 / 2}-z_{\min }\right) \sim 2 H / z_{1 / 2}$. Equation (5) then provides

$$
H=\left|\frac{n}{d n / d r}\right|=\frac{T}{(\mu g / k)+(d T / d r)} \sim \frac{z_{1 / 2}}{2} \Phi_{\min } .
$$

At the bottom of the temperature profile (stratosphere), $\mu \mathrm{g} / \mathrm{k}$ and $d T / d r$ are of the same order of magnitude. Consequently, increasing the value of the Pluto + Charon contribution to the light curve decreases the value of $\Phi$ (Figure 7), thus increasing the retrieved gradient $d T / d r$. This is illustrated in Figure 9 .

\section{B.3. Effect of Initial Conditions}

Once the density profile $n(r)$ is derived from the inversion, Equation (5) yields the temperature profile $T(r)$, provided a boundary condition $T_{\mathrm{b}}=T\left(\mathrm{r}_{\mathrm{b}}\right)$ is fixed at an arbitrary level $r_{\mathrm{b}}$. Let us consider two possible solutions $T(r)$ and $(T+\Delta T)(r)$ that differ by $\Delta T\left(r_{\mathrm{b}}\right)$ at $r_{\mathrm{b}}$, then expanding Equation (5) to first order in $(\Delta T / T)(r)$, we obtain:

$$
\frac{d}{d r}\left(\frac{\Delta T}{T}\right) \sim \frac{1}{H}\left(\frac{\Delta T}{T}\right)
$$

where we have approximated $H \sim k T / \mu g$. Thus, as $r$ increases, the relative difference $\Delta T / T$ diverge exponentially as:

$$
\left(\frac{\Delta T}{T}\right)(r) \sim\left(\frac{\Delta T}{T}\right)\left(r_{\mathrm{b}}\right) \cdot e^{\left(r-r_{\mathrm{b}}\right) / H} .
$$

This exponential divergence should not been confused with the one that is provided by Equation (14) for $\sigma_{T}$. The latter tends to zero as the noise tends to zero, while the former is inherent to the nature of Equation (5).

\section{REFERENCES}

Assafin, M., Camargo, J. I. B., Vieira Martins, R., et al. 2010, A\&A, 515, A32 Assafin, M., Campos, R. P., Vieira Martins, R., et al. 2008, P\&SS, 56, 1882 Assafin, M., Vieira-Martins, R., Braga-Ribas, F., et al. 2009, AJ, 137, 4046 Baum, W. A., \& Code, A. D. 1953, AJ, 58, 108

Benedetti-Rossi, G., Vieira Martins, R., Camargo, J. I. B., Assafin, M., \& Braga-Ribas, F. 2014, A\&A, 570, A86

Bosh, A. S., Person, M. J., Levine, S. E., et al. 2015, Icar, 246, 237

Boursier, C., Ménard, J., Doyennette, L., \& Menard-Bourcin, F. 2003, JPCA, 107,5280

Braga-Ribas, F., Sicardy, B., Ortiz, J. L., et al. 2013, ApJ, 773, 26

Brosch, N. 1995, MNRAS, 276, 571

Elliot, J. L., Ates, A., Babcock, B. A., et al. 2003, Natur, 424, 165

Elliot, J. L., Dunham, E. W., Bosh, A. S., et al. 1989, Icar, 77, 148

Elliot, J. L., Person, M. J., Gulbis, A. A. S., et al. 2007, AJ, 134, 1

Fray, N., \& Schmitt, B. 2009, P\&SS, 57, 2053

French, R. G., Toigo, A. D., Gierasch, P. J., et al. 2015, Icar, 246, 247

Girard, J. H., Kasper, M., Quanz, S. P., et al. 2010, Proc. SPIE, 7736E, 77362N

Gulbis, A. A. S., Emery, J. P., Person, M. J., et al. 2015, Icar, 246, 226

Hansen, C. J., \& Paige, D. A. 1996, Icar, 120, 247

Hubbard, W. B., Hunten, D. M., Dieters, S. W., Hill, K. M., \& Watson, R. D. 1988, Natur, 336, 452

Jehin, E., Gillon, M., Queloz, D., et al. 2011, Msngr, 145, 2

Krasnopolsky, V. A., \& Cruikshank, D. P. 1999, JGR, 104, 21979

Lara, L. M., Ip, W. H., \& Rodrigo, R. 1997, Icar, 130, 16

Lellouch, E. 1994, Icar, 108, 255

Lellouch, E., de Bergh, C., Sicardy, B., et al. 2015a, Icar, 246, 268

Lellouch, E., Gurwell, M., Butler, B., et al. 2015b, IAUC, 9273, 1

Lellouch, E., Laureijs, R., Schmitt, B., et al. 2000, Icar, 147, 220

Lellouch, E., Santos-Sanz, P., Lacerda, P., et al. 2013, A\&A, 557, A60

Lellouch, E., Sicardy, B., de Bergh, C., et al. 2009, A\&A, 495, 17

Lellouch, E., Stansberry, J., Emery, J., Grundy, W., \& Cruikshank, D. P. 2011, Icar, 214, 701

Lenzen, R., Hartung, M., Brandner, W., et al. 2003, Proc. SPIE, 4841, 944

Olkin, C. B., Young, L. A., Borncamp, D., et al. 2015, Icar, 246, 220

Olkin, C. B., Young, L. A., French, R. G., et al. 2014, Icar, 239, 15

Ortiz, J. L., Sicardy, B., Braga-Ribas, F., et al. 2012, Natur, 491, 566

Person, M. J., Dunham, E. W., Bosh, A. S., et al. 2013, AJ, 146, 83

Press, W. H., Teukolsky, S. A., Vetterling, W. T., \& Flannery, B. P. 1992, Numerical Recipes in C. The Art of Scientific Computing (New York: Cambridge Univ. Press)

Rousset, G., Lacombe, F., Puget, P., et al. 2003, Proc. SPIE, 4839, 140

Sicardy, B., Bellucci, A., Gendron, E., et al. 2006, Natur, 439, 52

Sicardy, B., Ferri, F., Roques, F., et al. 1999, Icar, 142, 357

Sicardy, B., Ortiz, J. L., Assafin, M., et al. 2011, Natur, 478, 493

Sicardy, B., Widemann, T., Lellouch, E., et al. 2003, Natur, 424, 168

Siddles, R., Wilson, G., \& Simpson, C. 1994, CPL, 225, 146

Strobel, D. F., Zhu, X., Summers, M. E., \& Stevens, M. H. 1996, Icar, 120, 266

Summers, M. E., Strobel, D. F., \& Gladstone, G. R. 1997, Chemical Models of Pluto's Atmosphere, ed. S. A. Stern \& D. J. Tholen (Tuscon, AZ: Univ. of Arizona Press), 391

Tholen, D. J., Buie, M. W., Grundy, W. M., \& Elliott, G. T. 2008, AJ, 135, 777 Vangvichith, M. 2013, Thèse de Doctorat, Ecole Polytechnique

Vapillon, L., Combes, M., \& Lecacheux, J. 1973, A\&A, 29, 135

Vuitton, V., Yelle, R. V., \& McEwan, M. J. 2007, Icar, 191, 722

Washburn, E. W. 1930, International Critical Tables of Numerical Data: Physics, Chemistry and Technology, Vol. 7 (New York: McGraw-Hill)

Yelle, R. V. 1991, AJ, 383, 380

Yelle, R. V., \& Lunine, J. I. 1989, Natur, 339, 288

Young, E. F., French, R. G., Young, L. A., et al. 2008, AJ, 136, 1757

Young, L. A. 2013, ApJL, 766, L22

Zacharias, N., Monet, D. G., Levine, S. E., et al. 2004, BAAS, 36, 1418

Zalucha, A. M., Gulbis, A. A. S., Zhu, X., Strobel, D. F., \& Elliot, J. L. 2011a, Icar, 211, 804

Zalucha, A. M., \& Michaels, T. I. 2013, Icar, 223, 819

Zalucha, A. M., Zhu, X., Gulbis, A. A. S., Strobel, D. F., \& Elliot, J. L. 2011b, Icar, 214, 685

Zhu, X., Strobel, D. F., \& Erwin, J. T. 2014, Icar, 228, 301 